\title{
Stability of \\ the In-phase Travelling Wave Solution in a Pair of Coupled Nerve Fibers
}

\section{Amitabha Bose E Christopher K. R. T. Jones}

\begin{abstract}
We consider the travelling wave that represents the simultaneous propagation of a voltage pulse along the length of two coupled nerve fibers. Conditions are given which guarantee the existence and stability of the wave. Two advances in the geometric techniques in the stability analysis of travelling waves are given: the first involves the Maslov Index, and the other the Exchange Lemma.
\end{abstract}

1. Introduction. Since the pioneering work of Hodgkin and Huxley, see [25], the propagation of impulses along nerve fibers has been modeled by a diffusion equation coupled with subsidiary ordinary differential equations. The nerve impulse itself is manifested as a solution of these equations that evolves by translating in the one-dimensional variable that represents distance along the nerve fiber. Such a solution is generally called a travelling wave and the application to neurophysiology has lent considerable motivation to the building of an extensive literature on travelling waves. Since the outside world is a ready supplier of imperfections, we can only expect travelling waves that are stable to perturbations in initial data to be physically observable. The stability of waves purporting to model physiological processes is thus of paramount importance. In this paper, we give conditions for the stability of a travelling wave that represents the inphase nerve impulse propagating along a pair of coupled, parallel nerve fibers. The problem is mathematically non-trivial on account of the relatively high dimensional equations resulting from having more than one fiber present. This mathematical challenge is met by invoking a few different approaches, including 
the use of the Maslov Index in locating eigenvalues of gradient systems, that occur on the fast time scale, and the Exchange Lemma which is used to gain accurate information on the transversality in the construction of the underlying wave in its ambient phase space; information which, since the work of Evans [1316], is known to be intimately related to stability. Part of the point of this work is then the development of techniques that are capable of extracting stability information about travelling waves in such a setting.

Various models of coupled nerve fibers have been proposed. The models due to Scott and Luzader [36] and Keener [30] are both based on the modeling of a single fiber by the FitzHugh-Nagumo system. In a certain popular limit, see Casten, Cohen and Lagerstrom [10], the variables evolve on different time scales. The fast variable represents the voltage difference across the membrane, while the slow variable, which has only an indirect physical interpretation even in the original Hodgkin-Huxley system, governs the recovery of the nerve fiber. We develop here a model which, in a natural way, generalizes both that of Keener and that of Scott and Luzader. The key feature of each of these models is that the coupling is reciprocal; in other words, the potential on the second fiber affects that on the first fiber in exactly the same way as it is itself affected by the first fiber. The nonlinear coupling then leads to a gradient nonlinearity for the system of partial differential equations governing the potentials.

The voltage on fiber $i$ is denoted by $u_{i}(i=1,2)$, and the recovery variable for fiber $i$ is denoted by $v_{i}$. We put the variables together to form a system governing $U=\left(u_{1}, u_{2}\right)$ and $V=\left(v_{1}, v_{2}\right)$ as

$$
\begin{aligned}
\varepsilon U_{t} & =\varepsilon^{2} D U_{x x}+f(U)-V \\
V_{t} & =\varepsilon(U-\gamma V)
\end{aligned}
$$

where $D$ is a diagonal, constant matrix, $f=\nabla F$ where $F: \mathbf{R}^{2} \rightarrow \mathbf{R}$ is a smooth function and $\gamma$ is a small, but fixed, parameter. The parameter $\varepsilon$ is assumed to be as small as needed. If we write out the nonlinear term in the first equation in coordinates, it can be seen how the coupling works. Indeed, in terms of $u_{1}$, $u_{2}$ and $f=\left(f_{1}, f_{2}\right)$ the coupling of fiber 1 to fiber 2 is determined by the partial derivative $\partial f_{1} / \partial u_{2}=\partial^{2} F / \partial u_{2} \partial u_{1}$, which, by the equality of mixed partials, is identical to $\partial f_{2} / \partial u_{1}=\partial^{2} F / \partial u_{1} \partial u_{2}$, which determines the coupling of fiber 2 to fiber 1 .

The model due to Keener [30] has exactly the form of (1) where

$$
f(U)=\left(\begin{array}{c}
g\left(u_{1}\right)+d\left(u_{2}-u_{1}\right) \\
g\left(u_{2}\right)+d\left(u_{1}-u_{2}\right)
\end{array}\right)
$$

and the function $g(u)=u(1-u)(u-a), 0<a<1 / 2$ being the usual cubic found in the FitzHugh-Nagumo model. The parameter $d$ is thought of as a coupling coefficient between the fibers. The condition of mixed partials is clearly satisfied, thus guaranteeing that the nonlinear term $f(U)=\nabla F(U)$ for some function $F(U)$, which can easily be written down. To reduce the Scott-Luzader model 
[36] to equation (1) requires a little more work. The equations have the form

$$
\begin{aligned}
\varepsilon U_{t} & =\varepsilon^{2} \Gamma U_{x x}+G(U)-V \\
V_{t} & =U-\gamma V
\end{aligned}
$$

where $G(U)=\left(g\left(u_{1}\right), g\left(u_{2}\right)\right)$, with $g(u)$ being the same cubic as used by Keener, and

$$
\Gamma=\left(\begin{array}{cc}
1-\alpha & \alpha \\
\alpha & 1-\alpha
\end{array}\right)
$$

so that the coupling on the diffusion is also reciprocal. Since the matrix $\Gamma$ is symmetric, it can be diagonalized by an orthogonal transformation $B$, i.e. $D=B^{T} \Gamma B$ is diagonal. If we then change variables by setting $U=B P$ and $V=B Q$ and multiply both equations in (2) on the left by $B^{T}$, the resulting equations for $(P, Q)$ have the form of (1).

The travelling wave we shall study in this paper is one in which both fibers fire simultaneously. In both the Keener and Scott-Luzader models this wave is identical on each fiber, i.e. $u_{1}=u_{2}$ and $v_{1}=v_{2}$, but in our more general model such a symmetric solution may not exist. The small parameter $\varepsilon$, however, allows us to separate the activation and recovery phases of the nerve pulses from each other. We thus force the two fibers to be firing simultaneously without requiring their being identical. Introducing a scaled time and space via $t=\varepsilon \tau$ and $x=\varepsilon y$, the equations (1) become

$$
\begin{aligned}
U_{\tau} & =D U_{y y}+f(U)-V \\
V_{\tau} & =\varepsilon(U-\gamma V) .
\end{aligned}
$$

Both the activation and recovery phases are governed by the $\varepsilon=0$ limit. In the activation phase $U$ will jump from 0 to $U_{+}(0)$, which is a steady state that has both components positive, so that both fibers have fired. This jump is achieved along a travelling wave (heteroclinic orbit) for the reduced fast system-see the next section for the details. The fast activation is then followed by a latent phase in which both $U$ and $V$ vary slowly, $V$ varying to a value $V^{*}$ and $U$ to $U_{+}\left(V^{*}\right)$. There is then a recovery phase in which $U$ jumps on a fast time scale to an unactivated state $U_{-}\left(V^{*}\right)$, after which both variables slowly return to rest.

This paper is organized as follows. In Section 2.1, we state conditions which will ensure existence of an in-phase travelling wave. Existence will be established by an appeal to a theorem due to Jones and Kopell [28]. In Section 2.2, we state additional conditions which will guarantee the stability of this wave. Stability of the wave is determined by locating the spectrum of an appropriate linear operator $L$. Other geometric constructs and theorems are discussed in Section 2 as well. Sections 3 and 4 are the main parts of the paper. In Section 3, we show how to use the Maslov Index to locate the eigenvalues of gradient 
systems. The Maslov Index is the generalization of Sturm-Liouville theory to such systems. It is an important fact that the eigenvalue equations of gradient systems preserve Lagrangian Planes. This fact allows us to restrict attention to the space of two-dimensional Lagrangian Planes, $\Lambda(2)$, which is a submanifold of the Grassmannian $G_{2,4}$. Our main result in this section is Lemma 3.7 which relates the index to the number of real eigenvalues of the linear operator. In Section 3.3, we apply the general theory to the fast travelling waves of our model as well as those of Keener's model. In Section 4, we use the Exchange Lemma in conjunction with the fact that $L$ has only two eigenvalues in some neighborhood of the origin (see Theorem 2.5 below) to finally determine stability of the wave. By translational invariance of travelling waves, one of the two eigenvalues must actually lie at the origin. The Exchange Lemma will then be used to compute the derivative of a certain analytic function which will locate the other.

2. Hypotheses and Theorems. We shall make various hypotheses concerning the system (1). These will guarantee that it possesses an appropriate travelling wave $(U(\xi), V(\xi))$, where $\xi=(x+\vartheta t) / \varepsilon$. This travelling wave will satisfy the system

$$
\begin{aligned}
U^{\prime} & =W \\
W^{\prime} & =\vartheta W-f(U)+V \\
V^{\prime} & =\frac{\varepsilon}{\vartheta}(U-\gamma V),
\end{aligned}
$$

where $^{\prime}=d / d \xi$. We seek then a trajectory of this latter system that is asymptotic to the rest state $U=V=0$, the existence of which is guaranteed by the hypotheses below.

2.1. Existence. Because of the smallness of $\varepsilon$, there are fast and slow time scales. The equations which govern the fast flow are obtained by analyzing (4) when $\varepsilon=0$ and are given by

$$
\begin{aligned}
U^{\prime} & =W \\
W^{\prime} & =\vartheta W-f(U)+V .
\end{aligned}
$$

The variable $V$ acts as a parameter in (5). The equation for the slow flow, which is obtained by introducing the rescaling $\eta=\varepsilon \xi$ into (4) and setting $\varepsilon=0$ is given by

$$
\begin{aligned}
0 & =-f(U)+V \\
\dot{V} & =\frac{1}{\vartheta}(U(V)-\gamma V),
\end{aligned}
$$

where ${ }^{\cdot}=\frac{d}{d \eta}$ and $U(V)$ is determined by solving the first equation of (6) for the variable $U$. The hypotheses required for existence of the wave are the following. 
(H1) There is a set $\Omega \subset R^{2}$ so that for every $V \in \Omega, V=f(U)$ has exactly three solutions, two of which, denoted $U_{ \pm}(V)$, are strict local maxima of $G(U, V)=F(U)-\langle V, U\rangle$, with $V$ fixed. In other words, $\operatorname{det} d^{2} F\left(U_{ \pm}(V)\right)>$ 0 and $\operatorname{tr} d^{2} F\left(U_{ \pm}(V)\right)<0$.

(H2) The point $(0,0) \in \Omega$ and $U_{-}(0)=0$, i.e. 0 is a rest state that is one of the strict local maxima.

(H3) The only solution of $V=f(U)$ and $U=\gamma V$ with $V \in \Omega$ is $U=V=0$.

Motivated by neurophysiological considerations, we are interested in two particular critical manifolds of (4). The first corresponds to the situation where both fibers are at rest and the second where both fibers are excited. Let $U_{-}=\left(u_{1_{-}}, u_{2_{-}}\right), U_{m}=\left(u_{1_{m}}, u_{2_{m}}\right)$ and $U_{+}=\left(u_{1_{+}}, u_{2_{+}}\right)$be the three solutions of $V=f(U)$ as described in (H1). For $i=1,2$, these can be ordered where $u_{i_{-}}<u_{i_{m}}<u_{i_{+}}$. We define manifold $(R)=\left\{(U, V): V=f(U), U=U_{-}(V)\right\}$ and manifold $(E)=\left\{(U, V): V=f(U), U=U_{+}(V)\right\}$.

(H4) When $\varepsilon=0$ there is a travelling wave (front) from $\left(U_{-}(0), 0,0\right)=(0,0,0)$ on manifold $(\mathrm{E})$ to $\left(U_{+}(0), 0,0\right)$ on manifold $(\mathrm{R})$ at speed $\vartheta^{*}>0$. Moreover, with $\vartheta^{\prime}=0$ appended to (4), the travelling wave exists as the transverse intersection of the center-unstable manifold of $\left(0,0, \vartheta^{*}\right)$ and the center-stable manifold of $\left(U_{+}(0), 0, \vartheta^{*}\right)$ in $(U, W, \vartheta)$ space.

(H5) There is a one-dimensional curve $C \subset \Omega$ so that for every $V^{*} \in C$ there is a travelling wave (back), when $\varepsilon=0$, from $\left(U_{+}\left(V^{*}\right), 0, V^{*}\right)$ on manifold (E) to $\left(U_{-}\left(V^{*}\right), 0, V^{*}\right)$ on manifold $(\mathrm{R})$ at speed $\vartheta^{*}$. In addition, this twodimensional family of travelling waves exists as the transverse intersection of the center-unstable manifold of $\left(U_{+}\left(V^{*}\right), 0, V^{*}\right)$ and the center-stable manifold of $\left(U_{-}\left(V^{*}\right), 0, V^{*}\right)$ in $(U, W, V)$ space.

(H6) For $\vartheta=\vartheta^{*}$, there exist two solutions to (6); the first connects $\left(U_{+}(0), 0\right)$ to $\left(U_{+}\left(V^{*}\right), V^{*}\right)$ with $U(V)=U_{+}(V)$ in (6), and the second connects $\left(U_{-}\left(V^{*}\right), V^{*}\right)$ to $(0,0)$ with $U(V)=U_{-}(V)$ in (6) for some $V^{*} \in C$.

Using the hypotheses above, we form a singular homoclinic orbit, $\Gamma_{\text {sing }}$, which consists of the travelling wave described in (H4), one of the waves of (H5), interspersed by the two solutions of the slow flow described in (H6). The hypotheses (H1)-(H6) are sufficient to allow us to apply the theorem due to Jones and Kopell [28] to establish the following theorem concerning existence and uniqueness of a real homoclinic orbit close to $\Gamma_{\text {sing }}$.

Theorem 2.1. There exists a locally unique travelling wave homoclinic solution $\tilde{U}(\xi)$ of $(4)$ which lies $O(\varepsilon)$ close to the singular homoclinic orbit $\Gamma_{\text {sing. }}$.

Local uniqueness means that it is the only wave within some neighborhood (in phase space) of itself. The goal of this paper is to give conditions under which this travelling wave is stable to perturbations in initial data. 
2.2. Stability. For the sake of completeness, we give a precise definition of stability. For this definition we recast (1) in a moving frame, i.e., in terms of the variables $\xi$ and $t$.

Definition 2.1. A travelling wave $\tilde{U}(\xi)$ is asymptotically stable if there is a neighborhood $N$ of $\tilde{U}(\xi)$ in $B U\left(\mathbf{R}, \mathbf{R}^{4}\right)$ (the space of bounded, uniformly continuous functions from $\mathbf{R}$ to $\left.\mathbf{R}^{4}\right)$ so that, if $\bar{U}(\xi, 0) \in N$ and $\bar{U}(\xi, t)$ satisfies (1), in a moving frame, then there is a $k$ for which $\|\bar{U}(\xi, t)-\tilde{U}(\xi+k)\|_{\infty} \rightarrow 0$ as $t \rightarrow \infty$.

In order to determine stability, we use the linearization of the PDE at $\tilde{U}(\xi)$, again in a moving frame.

$$
L\left(\begin{array}{c}
p_{1} \\
p_{2} \\
r_{1} \\
r_{2}
\end{array}\right)=\left(\begin{array}{c}
p_{1_{\xi \xi}}-\vartheta p_{1_{\xi}}+\frac{\partial^{2} F}{\partial u_{1}^{2}} p_{1}+\frac{\partial^{2} F}{\partial u_{2} \partial u_{1}} p_{2}-r_{1} \\
p_{2_{\xi \xi}}-\vartheta p_{2_{\xi}}+\frac{\partial^{2} F}{\partial u_{2} \partial u_{1}} p_{1}+\frac{\partial^{2} F}{\partial u_{2}^{2}} p_{2}-r_{2} \\
-\vartheta r_{1_{\xi}}+\varepsilon\left(p_{1}-\gamma r_{1}\right) \\
-\vartheta r_{2_{\xi}}+\varepsilon\left(p_{2}-\gamma r_{2}\right)
\end{array}\right) .
$$

The following proposition gives sufficient conditions for stability and can be concluded from results of Henry [24], or Bates and Jones [5].

Proposition 2.2. If the spectrum of $L$, denoted $\sigma(L)$, satisfies

(1) There is a $\beta<0$ so that $\sigma(L) \backslash\{0\} \subset\{\lambda: \operatorname{Re} \lambda<\beta\}$.

(2) 0 is a simple eigenvalue.

Then $\tilde{U}(\xi)$ is asymptotically stable.

Note that 0 must be an eigenvalue due to translation invariance. The hypotheses of Proposition 2.2 guarantee that the only neutral direction is that associated with translation. To assess the stability of $\tilde{U}(\xi)$, we need to determine the relevant properties of $\sigma(L)$ as prescribed by Proposition 2.2. The spectrum of $L$ splits into two parts: the point spectrum $\sigma_{p}(L)$, which is here defined to be the isolated eigenvalues of finite multiplicity, and the essential spectrum $\sigma_{e}(L)$, which is the rest. As in [26], the essential spectrum lies in the left half-plane bounded away from the imaginary axis, so it will cause no instabilities.

Lemma 2.3. (Jones [26]) If $\varepsilon>0$, there exists an $a<0$ such that $\sigma_{e}(L) \subset$ $\{\lambda: \operatorname{Re} \lambda<a\}$.

To locate $\sigma_{p}(L)$, we need two additional requirements which are tied to the gradient non-linearity. The condition $(\mathrm{H} 7)$ guarantees that the slow trajectory on manifold (E) transversely crosses the jump off set $C$ in the correct direction. 
(H1) The mixed partial derivative $\frac{\partial^{2} F}{\partial u_{2} \partial u_{1}}>0$.

(H2) The travelling wave front and back described in (H4) and (H5) each have Maslov Index 1.

The Maslov Index is the natural measure for stability of the travelling wave of the reduced system, i.e. for the front and the back. It will be seen in Section 3.2 that it is a property intrinsic to the phase space, and its use will be illustrated on the Keener model in Section 3.3.

Were we considering the problem of a single nerve fiber, the reduced system would be a scalar equation. Relative to this equation, the stability of the front solution could be resolved by an application of Sturm-Liouville theory, which would imply that the stability could be read off from the number of nodes of the wave. In the present context, however, the reduced equations are already a system and Sturm-Liouville theory is not applicable. This reduced system does, nevertheless, enjoy some specific structure which affords an application of a natural generalization of Sturm-Liouville theory. The structure comes from the fact that the nonlinear term is the gradient of a function. Such a situation occurs for variational problems and Morse, in his celebrated Index Theorem, see Milnor [32], proved that there is a connection between a certain index, which is related to the geometry of the solution in its ambient space, and the number of unstable directions for the variational problem. This index was shown by Arnol'd [3] to be the Maslov Index.

In our case, there is no direct variational structure. Nevertheless, as will be seen below, the Maslov Index can still be defined and bears a natural relationship to stability. In the variational context, (H8) means that the solution is minimum of the energy. If (4) is converted to a Hamiltonian system, using an integrating factor as done in Section 3.1 below, this interpretation of the Maslov Index can also be used in the current context.

Together, Proposition 2.2 and (H1)-(H8) will be used to establish the following theorem on the stability of the travelling wave $\tilde{U}(\xi)$.

Theorem 2.4. If $\varepsilon>0$ is sufficiently small, then $\tilde{U}(\xi)$ is stable relative to (1) in the sense of Definition 2.1.

For a scalar parabolic equation with a cubic non-linearity, which in Keener's model corresponds to one fiber with no recovery mechanism, Fife and McLeod [18] were the first to prove the stability of the travelling front. Evans [13-16] developed general techniques to study the spectrum of systems consisting of one scalar parabolic equation and a number of subsidiary ordinary differential equations, a class of equations he called "of nerve impulse type." He defined an analytic function $D(\lambda)$ whose zeroes correspond to the eigenvalues of the relevant linear operator. Jones [26] proved the stability of the "fast" travelling wave in the FitzHugh-Nagumo system, and a key technique in the proof involved using the Evans function. Yanagida [37] gave a later proof that used the Evans 
function even more extensively. Alexander, Gardner and Jones [1] generalized the construction of Evans' analytic function to systems of parabolic equations in their work which relates Chern numbers to eigenvalues. Dockery [12] has proved stability results for some travelling waves of Keener's 2-fiber model.

Denote by $Z^{\prime}=A Z$ the eigenvalue equation $(L-\lambda I) P=0$, which when written as a first order system is

$$
\begin{aligned}
& p_{1}^{\prime}=q_{1} \\
& p_{2}^{\prime}=q_{2} \\
& q_{1}^{\prime}=\vartheta q_{1}-\frac{\partial^{2} F}{\partial u_{1}^{2}} p_{1}-\frac{\partial^{2} F}{\partial u_{2} \partial u_{1}} p_{2}+r_{1}+\lambda p_{1}
\end{aligned}
$$

(8)

$$
\begin{aligned}
q_{2}^{\prime} & =\vartheta q_{2}-\frac{\partial^{2} F}{\partial u_{1} \partial u_{2}} p_{1}-\frac{\partial^{2} F}{\partial u_{2}^{2}} p_{2}+r_{2}+\lambda p_{2} \\
r_{1}^{\prime} & =\left(-\lambda r_{1}+\varepsilon\left(p_{1}-\gamma r_{1}\right)\right) / \vartheta \\
r_{2}^{\prime} & =\left(-\lambda r_{2}+\varepsilon\left(p_{2}-\gamma r_{2}\right)\right) / \vartheta .
\end{aligned}
$$

For $(8)$ at $\pm \infty$, the critical point $(0,0,0,0,0,0)$ has a two-dimensional unstable manifold and a four-dimensional stable manifold. From this, it follows that there exists solutions to $(8), X_{1}^{+}(\lambda, \xi), X_{2}^{+}(\lambda, \xi)$ which decay to 0 as $\xi \rightarrow-\infty$ and $X_{1}^{-}(\lambda, \xi), X_{2}^{-}(\lambda, \xi), X_{3}^{-}(\lambda, \xi), X_{4}^{-}(\lambda, \xi)$ which decay to 0 as $\xi \rightarrow+\infty$. Let $\Omega \supset\{\lambda: \operatorname{Re} \lambda>-b\}$ for some $b>0$.

Definition 2.2. The Evans function $D(\lambda)$ is defined by

$$
\begin{aligned}
& D(\lambda)=e^{-\int_{0}^{\xi} T R A(\lambda, \xi) d \xi} X_{1}^{+}(\lambda, \xi) \wedge X_{2}^{+}(\lambda, \xi) \\
& \wedge X_{1}^{-}(\lambda, \xi) \wedge X_{2}^{-}(\lambda, \xi) \wedge X_{3}^{-}(\lambda, \xi) \wedge X_{4}^{-}(\lambda, \xi)
\end{aligned}
$$

for $\lambda \in \Omega$ with values in $\bigwedge^{6}\left(\mathbf{C}^{\mathbf{6}}\right)$.

Using Abel's formula, it can be shown that $D(\lambda)$ is in fact independent of $\xi$. The Evans function enjoys the following properties:

(1) $D(\lambda)$ is analytic in $\lambda \in \Omega$.

(2) $D(\lambda)=0$ if and only if $\lambda$ is an eigenvalue of $L$.

(3) The algebraic multiplicity of an eigenvalue equals the order of the zero of $D(\lambda)$.

Properties (1) and (2) follow directly from [1]. The third follows from an argument similar to one found in [20]. 
In Sections 2.3 and 3 below, we prove that the front and back are stable relative to their partial differential equations. Using this information, together with arguments similar to those in Jones [26] and Alexander, Gardner and Jones [1], it can be shown that the eigenvalues of the linearization at the full wave are approximated by those associated with the fast reduced systems, which establishes the following theorem.

Theorem 2.5. There exists a simple closed curve $K$ which encloses the origin and all of the eigenvalues of $L$ in the right half-plane. Moreover if $(\mathbf{H 1})-$ (H8) are satisfied, then $L$ possesses exactly two eigenvalues within $K$.

The idea of Theorem 2.5 is that each stable fast jump provides exactly one eigenvalue near to the origin. One of these, by translational invariance, lies at the origin. We will locate the second eigenvalue by using the Exchange Lemma to find the sign of the derivative of $D(\lambda)$ at $\lambda=0$, and from this conclude Theorem 2.4 .

2.3. Stability of the singular in-phase front. Setting $\varepsilon=0$, the singular limit of the travelling wave decomposes into two fast heteroclinic jumps. The front solution connects manifolds $\mathrm{R}$ and $\mathrm{E}$. Thus we call it the $\mathrm{R}-\mathrm{E}$ travelling wave front. In this section we discuss the stability of this wave with respect to the relevant reduced partial differential equation. There are corresponding results for the $\mathrm{E}-\mathrm{R}$ back wave.

Consider the equations for the front recast in a moving frame where we have set $v_{1}=v_{2}=0$.

$$
\begin{aligned}
& u_{1_{t}}=u_{1_{\xi \xi}}-\vartheta u_{1_{\xi}}+f_{1}\left(u_{1}, u_{2}\right), \\
& u_{2_{t}}=u_{2_{\xi \xi}}-\vartheta u_{2_{\xi}}+f_{2}\left(u_{1}, u_{2}\right) .
\end{aligned}
$$

Linearize these equations around the front solution $U_{F}(\xi)$, which exists at some wave speed $\vartheta=\vartheta^{*}$,

$$
L_{F}\left(\begin{array}{c}
p_{1} \\
p_{2}
\end{array}\right)=\left(\begin{array}{c}
p_{1_{\xi \xi}}-\vartheta^{*} p_{1_{\xi}}+\frac{\partial^{2} F}{\partial u_{1}^{2}} p_{1}+\frac{\partial^{2} F}{\partial u_{2} \partial u_{1}} p_{2} \\
p_{2_{\xi \xi}}-\vartheta^{*} p_{2_{\xi}}+\frac{\partial^{2} F}{\partial u_{1} \partial u_{2}} p_{1}+\frac{\partial^{2} F}{\partial u_{2}^{2}} p_{2}
\end{array}\right)
$$

where $L_{F}: B U\left(\mathbf{R}, \mathbf{R}^{\mathbf{2}}\right) \rightarrow B U\left(\mathbf{R}, \mathbf{R}^{\mathbf{2}}\right)$.

Theorem 2.6. If $(\mathbf{H 8})$ is satisfied, then $U_{F}(\xi)$ is stable relative to (10) in the sense of Definition 2.1.

The essential spectrum of $L_{F}$ lies in the left half-plane bounded away from the imaginary axis. The Evans function associated with $L_{F}$, which we call $D_{F}(\lambda)$, 
is defined similarly to $D(\lambda)$. To prove the stability of the front we need the following properties for $D_{F}(\lambda)$ to hold.

(1) $D_{F}(0)=0$.

(2) $D_{F}(\lambda) \neq 0$ for $\lambda \in\{\lambda: \operatorname{Re} \lambda>b\}$ for some $b<0$ except at $\lambda=0$.

(3) 0 is a simple eigenvalue of $D_{F}(\lambda)$.

By translational properties of travelling waves, $\lambda=0$ is an eigenvalue, which implies (1) is true. We next show statement (3).

Consider equation (5) with $v_{1}=v_{2}=0$ and the equation $\vartheta^{\prime}=0$ appended.

$$
\begin{aligned}
u_{1}^{\prime} & =w_{1} \\
w_{1}^{\prime} & =\vartheta w_{1}-f_{1}\left(u_{1}, u_{2}\right) \\
u_{2}^{\prime} & =w_{2} \\
w_{2}^{\prime} & =\vartheta w_{2}-f_{2}\left(u_{1}, u_{2}\right) \\
\vartheta^{\prime} & =0 .
\end{aligned}
$$

It follows from hypothesis (H1) that the critical point $(0,0,0,0, \vartheta)$ has a threedimensional center-unstable manifold $W_{F}^{c u}(0)$ and the critical point $\left(u_{1_{+}}, 0, u_{2_{+}}, 0, \vartheta\right)$ also has a three-dimensional center-stable manifold $W_{F}^{c s}\left(U_{+}(0)\right)$. Using hypothesis (H4), the solution $U_{F}(\xi)$ exists as the transverse intersection of $W_{F}^{c u}(0)$ and $W_{F}^{c s}\left(U_{+}(0)\right)$. Alexander and Jones [2] show that the transversality of the these manifolds is equivalent to simpleness of the translational eigenvalue, thus proving (3).

To prove statement (2), we will use the Maslov Index to locate the possible eigenvalues of $L_{F}$ in the right half-plane. The transversality of $U_{F}(\xi)$ and an application of Corollary 3.8 below will then prove Theorem 2.6 .

3. Eigenvalues of a gradient system. In this section we will prove that $L_{F}$ has no eigenvalues in the open right half-plane if $U_{F}$ has Maslov Index 1. The Maslov Index provides a means for finding real eigenvalues of operators associated with gradient systems. The index counts the number of winds that a certain curve of Lagrangian Planes makes in a sub-manifold of projective space. In this section we develop a general theory for finding eigenvalues of gradient systems.

In the Sturm-Liouville theory, the presence of eigenvalues can be determined by studying an angular variable. The Sturm-Liouville theory applies only to scalar second order differential equations, for which there exists a natural definition of the angular variable. Converting the second order equation to a system of two first order equations, the angular variable is defined as the arctangent of the ratio of the two ensuing variables. In the present situation, we have two second order equations and thus a system of four first order equations. As a result, it is not immediately clear how to define a useful angular variable and also whether it will have any relevance to the existence of eigenvalues. The appropriate way to 
formulate such is to define the variable as a projection from the set of Lagrangian planes to the circle $S^{1}$.

We consider systems of the form

$$
u_{t}=u_{x x}+f(u),
$$

where $u \in \mathbf{R}^{2}$ and $x, t \in \mathbf{R}$. We require that $f(u)$ be conservative. Thus there exists some scalar valued function $F(u) \in C^{2}$ such that $\nabla F(u)=f(u)$, i.e. if $f(u)=\left(f_{1}\left(u_{1}, u_{2}\right), f_{2}\left(u_{1}, u_{2}\right)\right)$, then $\partial F / \partial u_{1}=f_{1}$ and $\partial F / \partial u_{2}=f_{2}$. Introducing the change of variable $\xi=x+\vartheta t$ into (13), and writing the resulting equations as a first order system, we obtain

$$
\begin{aligned}
u_{1}^{\prime} & =w_{1} \\
u_{2}^{\prime} & =w_{2} \\
w_{1}^{\prime} & =\vartheta w_{1}-f_{1}\left(u_{1}, u_{2}\right) \\
w_{2}^{\prime} & =\vartheta w_{2}-f_{2}\left(u_{1}, u_{2}\right) .
\end{aligned}
$$

We make the following three assumptions on the reaction term $f(u)$ and on (14).

(a) There exist values $\left(u_{1_{ \pm}}, u_{2_{ \pm}}\right)$such that $f_{i}\left(u_{1_{ \pm}}, u_{2_{ \pm}}\right)=0$ for $i=1,2$.

(b) The critical points $\left(u_{1_{ \pm}}, u_{2_{ \pm}}, 0,0\right)$ are both hyperbolic for (14) and have two-dimensional stable and unstable manifolds.

(c) There exists a travelling wave solution, $U_{G}(\xi)$, to (14) which tends to $\left(u_{1_{ \pm}}, u_{2_{ \pm}}, 0,0\right)$ exponentially fast as $\xi \rightarrow \pm \infty$ at some known wave speed $\vartheta$.

As before, we recast (13) into a moving frame, linearize the equations in question about the travelling wave solution $U_{G}(\xi)$, and obtain the resulting operator

$$
L_{G} P=\left(\begin{array}{c}
p_{1}^{\prime \prime}-\vartheta p_{1}^{\prime}+\frac{\partial f_{1}}{\partial u_{1}} p_{1}+\frac{\partial f_{1}}{\partial u_{2}} p_{2} \\
p_{2}^{\prime \prime}-\vartheta p_{2}^{\prime}+\frac{\partial f_{2}}{\partial u_{1}} p_{1}+\frac{\partial f_{2}}{\partial u_{2}} p_{2}
\end{array}\right) .
$$

Writing the eigenvalue equation $\left(L_{G}-\lambda I\right) P=0$ as a first order system, we obtain

$$
\begin{aligned}
& p_{1}^{\prime}=p_{3} \\
& p_{2}^{\prime}=p_{4} \\
& p_{3}^{\prime}=\vartheta p_{3}-\frac{\partial f_{1}}{\partial u_{1}} p_{1}-\frac{\partial f_{1}}{\partial u_{2}} p_{2}+\lambda p_{1} \\
& p_{4}^{\prime}=\vartheta p_{4}-\frac{\partial f_{2}}{\partial u_{1}} p_{1}-\frac{\partial f_{2}}{\partial u_{2}} p_{2}+\lambda p_{2} .
\end{aligned}
$$


Note that the assumptions above imply (16) is asymptotically autonomous, and that the unstable and stable subspaces of the origin at $\pm \infty$, denoted $W_{ \pm}^{u}$ and $W_{ \pm}^{s}$ respectively, are also two-dimensional. The parameter $\lambda$ will be an eigenvalue if $W_{-}^{u} \cap W_{+}^{s} \neq\{\varnothing\} \times(-\infty,+\infty)$, i.e. if the intersection of these two subspaces is at least one-dimensional, for then there is a bounded solution of (16) at that value of $\lambda$.

3.1. Gradient systems have real eigenvalues. We show here that for gradient systems, the point spectrum of $L_{G}$ must be real.

Lemma 3.1. The point spectrum $\sigma_{p}\left(L_{G}\right) \subset \mathbf{R}$.

Proof. It is a standard fact that when $\vartheta=0, L_{G}$ is self-adjoint in $L^{2}$, since then $L_{G}$ has no first derivative term. Using a transformation as in Sattinger [35] to eliminate the first derivative for $\vartheta \neq 0$, define a new operator $M$ by $M=e^{-\vartheta \xi / 2} L_{G} e^{\vartheta \xi / 2}$. It is not difficult to show that

$$
M P=\left(\begin{array}{c}
p_{1}^{\prime \prime}-\frac{\vartheta^{2}}{4} p_{1}+\frac{\partial f_{1}}{\partial u_{1}} p_{1}+\frac{\partial f_{1}}{\partial u_{2}} p_{2} \\
p_{2}^{\prime \prime}-\frac{\vartheta^{2}}{4} p_{2}+\frac{\partial f_{2}}{\partial u_{1}} p_{1}+\frac{\partial f_{2}}{\partial u_{2}} p_{2}
\end{array}\right),
$$

from which it easily follows that $M$ is self-adjoint in $L^{2}$. Finally, it is not difficult to prove that $\lambda$ is an eigenvalue of $M$ if and only if it is an eigenvalue of $L_{G}$, in $L^{2}$. Furthermore, the eigenvalues of $L_{G}$ in $L^{2}$ coincide with those of $L_{G}$ in $B U\left(\mathbf{R}, \mathbf{R}^{2}\right)$.

3.2. The Maslov Index. In this section, we show how to use the Maslov Index to count the number of real eigenvalues in the interval $[0, \infty)$ for gradient systems. Arnol'd [3] gives an expression for this index in terms of the Cayley transform. We will give a new representation for the index in terms of Plücker coordinates.

The Maslov Index is an index of a curve of Lagrangian planes.

Definition 3.2. A Lagrangian plane $\Psi$ is a two-dimensional subspace of $\mathbf{R}^{4}$ that satisfies $\left(y_{1}, J y_{2}\right)=0$ for all $y_{1}, y_{2} \in \Psi$, where

$$
J=\left[\begin{array}{cc}
0 & -I \\
I & 0
\end{array}\right]
$$

is the symplectic matrix and $I$ is the $2 \times 2$ identity matrix. 
Let $y_{1}=\left(a_{1}, a_{2}, a_{3}, a_{4}\right), y_{2}=\left(b_{1}, b_{2}, b_{3}, b_{4}\right)$, then $\left(y_{1}, J y_{2}\right)=\left(a_{1} b_{3}-b_{1} a_{3}\right)+$ $\left(a_{2} b_{4}-b_{2} a_{4}\right)$. The Plücker coordinate $P_{i j}$, of the plane spanned by $y_{1}$ and $y_{2}$, is defined to be the $2 \times 2$ subdeterminant of the $i$ th and $j$ th column of the $2 \times 4$ matrix formed by taking $y_{1}$ and $y_{2}$ as the rows. The set of numbers $P_{i j}$ are the Plücker coordinates if the following Grassmannian condition is satisfied.

$$
\text { Grassmannian: } P_{12} \cdot P_{34}-P_{13} \cdot P_{24}+P_{14} \cdot P_{23}=0 \text {. }
$$

Also, $P_{13}=a_{1} b_{3}-b_{1} a_{3}$, and $P_{24}=a_{2} b_{4}-a_{4} b_{2}$. Thus $\left(y_{1}, J y_{2}\right)=P_{13}+P_{24}$. This proves $\Lambda(2) \subset G_{2,4}$ is given by

$$
\text { Lagrangian Plane: } P_{13}+P_{24}=0 \text {. }
$$

A convenient representation of $\Lambda(2)$ is based on matrix groups. It turns out that $\Lambda(2) \cong U(2) / O(2)$, where $U(2)$ is the group of $2 \times 2$ unitary matrices and $O(2)$ is the subgroup of real orthogonal $2 \times 2$ matrices. Since the determinant of an orthogonal transformation is either \pm 1 , the map Det ${ }^{2}$ acts as a projection onto $S^{1}$, i.e. $\operatorname{Det}^{2}: \Lambda(2) \rightarrow S^{1}$. Both Arnol'd and Jones show that $\Lambda(2)$ is a fiber bundle over the base space $S^{1}$ with fiber $S^{2}$ and clutching function the antipodal map. The space of Lagrangian planes has fundamental group $\mathbf{Z}$. Thus the mapping Det $^{2}$, which can be used to count the number of times the image of $\Lambda(2)$ circles $S^{1}$, provides a mechanism to determine how many winds certain Lagrangian planes undergo in a sub-manifold of projective phase space. This winding number is the Maslov Index.

For every $w \in \Lambda(2)$, spanned by $y_{1}$ and $y_{2}$ which has $P_{12} \neq 0$, there exists a transformation $\phi \in \mathrm{GL}(2)$ such that $\phi w=(I \mid S)$, where I is the $2 \times 2$ identity matrix and $\mathrm{S}$ is a $2 \times 2$ symmetric matrix. The identification above is

$$
\phi\left(\begin{array}{llll}
a_{1} & a_{2} & a_{3} & a_{4} \\
b_{1} & b_{2} & b_{3} & b_{4}
\end{array}\right)=\left(\begin{array}{cccc}
1 & 0 & s_{1} & s \\
0 & 1 & s & s_{4}
\end{array}\right) .
$$

This identification serves to define local coordinates on the set $P_{12} \neq 0$. Arnol'd [3] shows that

$$
\operatorname{Det}^{2} w=\operatorname{det} \frac{I-i S}{I+i S}=\operatorname{det}(I-i S)(I+i S)^{-1},
$$

which is the Cayley transform.

We now give a new representation of the Cayley transform using Plücker coordinates. This will be done in the local patch $P_{12} \neq 0$. But the final formula (22) will hold on all of $\Lambda(2)$. The map $\operatorname{Det}^{2} w=\operatorname{det} I-i S / I+i S$ can be written as

$$
\operatorname{det}(I-i S)(I+i S)^{-1}=\frac{\left(1+s_{4}\right)^{2}\left(1+s_{1}\right)^{2}+s^{4}+2 s^{2}\left(1-s_{1} s_{4}\right)}{\left(1-s_{1} s_{4}+s^{2}+i\left(s_{1}+s_{4}\right)\right)^{2}} .
$$


To determine how many times a trajectory winds around $S^{1}$, we calculate the angular equation associated with the map Det $^{2}$, using the arctangent.

$$
\kappa=\tan ^{-1} \operatorname{Im} \operatorname{Det}^{2} / \operatorname{Re} \operatorname{Det}^{2}
$$

Since the numerator of $(20)$ is strictly real, it is not hard to show that

$$
\kappa=\tan ^{-1} \frac{-2\left(s_{1}+s_{4}\right)\left(1-s_{1} s_{4}+s^{2}\right)}{\left(1-s_{1} s_{4}+s^{2}\right)^{2}-\left(s_{1}+s_{4}\right)^{2}} .
$$

The $2 \times 4$ matrix of (19) from which we defined $\operatorname{Det}^{2}$ is already in normalized form. Thus, we have $\hat{P}_{12}=1, \hat{P}_{13}=s, \hat{P}_{24}=-s, \hat{P}_{14}=s_{4}, \hat{P}_{23}=-s_{1}, \hat{P}_{34}=$ $s_{1} s_{4}-s^{2}$. The ${ }^{\wedge}$ connotes a normalized form. In this case the 2 -form $P_{12}$ has been used to normalize, i.e. $\hat{P}_{i j}=P_{i j} / P_{12}$. In these coordinates, we see that

$$
\begin{aligned}
s=\frac{P_{13}}{P_{12}}, & s_{1}=\frac{-P_{23}}{P_{12}}, \\
s_{4}=\frac{P_{14}}{P_{12}}, & s_{1} s_{4}-s^{2}=\frac{P_{34}}{P_{12}} .
\end{aligned}
$$

Substituting into (21) and rearranging terms, we obtain

$$
\kappa=\tan ^{-1} \frac{-2\left(P_{14}-P_{23}\right)\left(P_{12}-P_{34}\right)}{\left(P_{12}-P_{34}\right)^{2}-\left(P_{14}-P_{23}\right)^{2}} .
$$

This is the expression for the angular variable in terms of Plücker coordinates. We note that $\kappa$ is a function of $\xi$ and of all the parameters of the problem, in particular of $\lambda$.

Evolution equations for the $P_{i j}$ 's are obtained using the product rule, $P_{i j}^{\prime}=$ $p_{i} \wedge p_{j}^{\prime}+p_{i}^{\prime} \wedge p_{j}$ and (16). It is an important fact that such equations preserve (17) and (18) for the trajectories of interest. The evolution equations for the six independent 2 -forms are

$$
\begin{aligned}
& P_{12}^{\prime}=P_{14}-P_{23} \\
& P_{13}^{\prime}=\vartheta P_{13}-\frac{\partial f_{1}}{\partial u_{2}} P_{12} \\
& P_{24}^{\prime}=\vartheta P_{24}+\frac{\partial f_{2}}{\partial u_{1}} P_{12} \\
& P_{14}^{\prime}=\vartheta P_{14}+\left(\lambda-\frac{\partial f_{2}}{\partial u_{2}}\right) P_{12}+P_{34}
\end{aligned}
$$




$$
\begin{aligned}
& P_{23}^{\prime}= \vartheta P_{23}-\left(\lambda-\frac{\partial f_{1}}{\partial u_{1}}\right) P_{12}-P_{34} \\
& P_{34}^{\prime}=2 \vartheta P_{34}+\left(\lambda-\frac{\partial f_{1}}{\partial u_{1}}\right) P_{14}-\left(\lambda-\frac{\partial f_{2}}{\partial u_{2}}\right) P_{23} \\
& \\
& \quad+\frac{\partial f_{2}}{\partial u_{1}} P_{13}-\frac{\partial f_{1}}{\partial u_{2}} P_{24} .
\end{aligned}
$$

It is not obvious that (23) preserves Lagrangian planes due to the presence of $\vartheta$. We next verify this. Using the gradient condition $\partial f_{1} / \partial u_{2}=\partial f_{2} / \partial u_{1}$, we obtain $\left(P_{13}+P_{24}\right)^{\prime}=\vartheta\left(P_{13}+P_{24}\right)$. Since $P_{13}+P_{24}=0$ for a Lagrangian plane, and in particular at $-\infty$, it follows that $\left(P_{13}+P_{24}\right)^{\prime}=0$, which implies $P_{13}+P_{24} \equiv 0$. Thus (18) holds.

We are interested in determining the number of winds around $S^{1}$ of a given Lagrangian plane. This is achieved by computing an evolution equation for the angular variable $\kappa(\xi)$ by differentiating (22) with respect to $\xi$. That is,

$$
\kappa^{\prime}=\frac{-2\left(\left(P_{12}-P_{34}\right)\left(P_{14}^{\prime}-P_{23}^{\prime}\right)-\left(P_{12}^{\prime}-P_{34}^{\prime}\right)\left(P_{14}-P_{23}\right)\right)}{\left(P_{12}-P_{34}\right)^{2}+\left(P_{14}-P_{23}\right)^{2}} .
$$

There are two important facts about gradient systems. First, $\kappa$ is monotone decreasing in the eigenvalue parameter $\lambda$ for each $\xi$. Second, as $\lambda$ tends to $+\infty$, $(\partial / \partial \xi) \kappa(\lambda, \xi)$ tends to zero. We state these two facts precisely in the following lemmas. In what follows, let $\kappa(\lambda, \xi)=\kappa_{\lambda}(\xi)$.

Lemma 3.3. For each $\xi$, if $\lambda_{1}<\lambda_{2}$, then $\kappa_{\lambda_{1}}(\xi)>\kappa_{\lambda_{2}}(\xi)$.

Proof. For convenience denote $\rho(\xi)=\kappa_{\lambda_{1}}(\xi)-\kappa_{\lambda_{2}}(\xi)$. We will show that, whenever $\rho(\xi)=0, \rho^{\prime}(\xi)>0$. Suppress dependence on $\xi$ and assume that $\rho=0$. Then, a rather tedious calculation involving the arctangent, arcsine and arccosine implies that $P_{i j_{\lambda_{1}}}=P_{i j_{\lambda_{2}}}$, where the $P_{i j}$ 's correspond to the six 2 -forms of (23). We will drop the subscript $\lambda_{i}$ wherever $P_{i j_{\lambda_{1}}}=P_{i j_{\lambda_{2}}}$. Call $Q=\left(P_{12}-P_{34}\right)^{2}+\left(P_{14}-P_{23}\right)^{2}$. Note that $Q>0$. Using (24), we obtain

$$
\begin{aligned}
\rho^{\prime}=\frac{-2}{Q}( & \left(P_{14_{\lambda_{1}}}-P_{14_{\lambda_{2}}}\right)^{\prime}\left(P_{12}-P_{34}\right)-\left(P_{14}-P_{23}\right)\left(P_{12_{\lambda_{1}}}-P_{12_{\lambda_{2}}}\right)^{\prime} \\
& \left.-\left(P_{23_{\lambda_{1}}}-P_{23_{\lambda_{2}}}\right)^{\prime}\left(P_{12}-P_{34}\right)+\left(P_{14}-P_{23}\right)\left(P_{34_{\lambda_{1}}}-P_{34_{\lambda_{2}}}\right)^{\prime}\right) .
\end{aligned}
$$

Now $P_{14_{\lambda_{1}}}=P_{14_{\lambda_{2}}}, P_{12_{\lambda_{1}}}=P_{12_{\lambda_{2}}}, P_{34_{\lambda_{1}}}=P_{34_{\lambda_{2}}}$ and $P_{23_{\lambda_{1}}}=P_{23_{\lambda_{2}}}$ implies 


$$
\begin{aligned}
& P_{14_{\lambda_{1}}}^{\prime}-P_{14_{\lambda_{2}}}^{\prime}=\left(\lambda_{1}-\lambda_{2}\right) P_{12} \quad, \quad P_{12_{\lambda_{1}}}^{\prime}-P_{12_{\lambda_{2}}}^{\prime}=0 \\
& P_{23_{\lambda_{1}}}^{\prime}-P_{23_{\lambda_{2}}}^{\prime}=\left(\lambda_{2}-\lambda_{1}\right) P_{12} \quad, \quad P_{34_{\lambda_{1}}}^{\prime}-P_{34_{\lambda_{2}}}^{\prime}=\left(\lambda_{1}-\lambda_{2}\right)\left(P_{14}-P_{23}\right) \text {. }
\end{aligned}
$$

With the help of the Grassmannian condition, (17), this implies

$$
\begin{aligned}
\rho^{\prime} & =\frac{-2}{Q}\left(\lambda_{1}-\lambda_{2}\right)\left(2\left(P_{12}\left(P_{12}-P_{34}\right)+\left(P_{14}-P_{23}\right)^{2}\right)\right. \\
& =\frac{-2}{Q}\left(\lambda_{1}-\lambda_{2}\right)\left(2 P_{12}^{2}+2 P_{13}^{2}+P_{14}^{2}+P_{23}^{2}\right) .
\end{aligned}
$$

Since $\lambda_{1}<\lambda_{2}$, we see that $\rho^{\prime}>0$. Thus, whenever $\rho=0, \rho^{\prime}>0$.

It is also true that $\kappa_{\lambda_{1}}(-\infty)>\kappa_{\lambda_{2}}(-\infty)$. This is shown by using the unstable eigenvectors. The calculations are straightforward, but long and tedious, so we omit them. This implies $\kappa$ is strictly decreasing in $\lambda$.

Lemma 3.4. As $\lambda \rightarrow+\infty,(\partial / \partial \xi) \kappa(\lambda, \xi) \rightarrow 0$.

Proof. Introduce the rescaling $\sqrt{\lambda} \zeta=\xi$ into the eigenvalue problem $L_{G} P=$ $\lambda P$. Using (24), it can be seen that $(\partial / \partial \xi) \kappa(\lambda, \xi)$ has a limit as $\lambda \rightarrow+\infty$. Passing to this limit, we obtain the following system of first order equations, where ${ }^{\cdot}=d / d \zeta$ :

$$
\begin{array}{ll}
\dot{p}_{1}=q_{1} & \dot{p}_{2}=q_{2} \\
\dot{q}_{1}=p_{1} & \dot{q}_{2}=p_{2} .
\end{array}
$$

These equations are Hamiltonian so they preserve Lagrangian planes. The six 2 -forms associated with (25) are

$$
\begin{array}{ll}
\dot{P}_{12}=P_{14}-P_{23} & \dot{P}_{13}=0 \\
\dot{P}_{14}=P_{34}+P_{12} & \dot{P}_{24}=0 \\
\dot{P}_{34}=P_{14}-P_{23} & \dot{P}_{23}=-P_{34}-P_{12} .
\end{array}
$$

Since $\dot{P}_{12}-\dot{P}_{34}=0$,

$$
\dot{\kappa}=\frac{-4\left(P_{12}^{2}-P_{34}^{2}\right)}{\left(P_{12}-P_{34}\right)^{2}+\left(P_{14}-P_{23}\right)^{2}} .
$$

At $-\infty, 0$ has a two-dimensional $\mathrm{W}^{u}$. The vectors $(1,1,-1,-1)$ and $(1,-1,1,-1)$ span the tangent subspace of $W^{u}$ at $-\infty$. Thus $P_{12}=-2$ and $P_{34}=2$. Therefore $\dot{\kappa}=0$. Thus it can be seen that $(\partial / \partial \xi) \kappa(\lambda, \xi) \rightarrow 0$. 
Let $\psi \in \Lambda(2)$. Arnol'd [4] introduces the notion of the train of $\psi$. Jones [27] gives an equivalent definition.

Definition 3.5. The train of $\psi \in \Lambda(2)$, denoted $\mathcal{D}(\psi)$, is the set of all $\phi \in \Lambda(2)$ so that (as two-dimensional subspaces of $\left.\mathbf{R}^{4}\right) \phi \cap \psi \neq\{0\}$. I.e., it is the set of all subspaces that intersect the given one non-trivially. The point $\psi$ is called the vertex of the train.

To understand more clearly the asymptotic behavior of certain solutions, it is helpful to compactify the $\xi$ variable with a new variable $\tau$ via the relation

$$
\xi=\frac{1}{2 \delta} \ln \frac{1+\tau}{1-\tau} .
$$

Using $\tau$ as a dependent variable, (16) can now be rewritten as

$$
\begin{aligned}
Y^{\prime} & =A(\lambda, \tau) Y, \\
\tau^{\prime} & =\delta\left(1-\tau^{2}\right)
\end{aligned}
$$

where ${ }^{\prime}=d / d \xi$. Now, $\tau= \pm 1$ carries the asymptotic flow at $\xi= \pm \infty$. If $\delta$ is chosen small enough, then (27) is $C^{1}$ on $\mathbf{C}^{4} \times[-1,+1]$; see [1].

We now determine representatives in $\Lambda(2)$ for $W_{-}^{u}$ and $W_{+}^{s}$. Consider the restriction of $W_{-}^{u}$ to a particular slice of $\tau$. Let $Z^{u}(\lambda, \tau)=W_{-}^{u} \cap\{\tau\}$. Let $\Phi(\lambda, \tau)=\sigma(Z(\lambda, \tau))$ where $\sigma: \mathbf{R}^{4} \times[-1,+1] \rightarrow \mathbf{R}^{4}$ is the natural projection. If $Q(\lambda, \tau)=P_{1}(\lambda, \tau) \wedge P_{2}(\lambda, \tau)$, where $P_{1}$ and $P_{2}$ are solutions that span $\Phi(\lambda, \tau)$, then $Q$ is a curve of one-dimensional subspaces in $\Lambda^{2}\left(\mathbf{R}^{4}\right)$. Let $\Pi: \mathbf{R}^{6} \backslash\{0\} \rightarrow$ $\mathbf{R} P^{5}$. Then $\Pi(Q)=\tilde{Q}$. Set $\phi(\lambda, \tau)=\tilde{Q}(\lambda, \tau)$. Finally the curve $\zeta(\lambda, \tau)=$ $(\phi(\lambda, \tau), \tau)$ is in $G_{2,4} \times[-1,+1]$.

Similarly, $Z^{s}(\lambda, \tau)=W_{+}^{s} \cap\{\tau\}$. Let $Z^{1}=Z^{s}(\lambda,+1)$, the stable subspace of 0 inside $\tau=+1$. The train of $\sigma\left(Z^{1}\right)$ is

$$
\begin{gathered}
\mathcal{D}\left(\sigma\left(Z^{1}\right)\right)=\{\psi \in \Lambda(2): \text { the subspace } \Psi \text { determined by } \psi \text { satisfies } \\
\left.\Psi \cap \sigma\left(Z^{1}\right) \neq\{0\}\right\} .
\end{gathered}
$$

Now $\lambda$ is an eigenvalue if $\omega\left(\phi\left(\lambda, \tau_{0}\right), \tau_{0}\right) \cap \mathcal{D}\left(\sigma\left(Z^{1}\right)\right) \times\{+1\} \neq \varnothing$.

We seek a relationship between the covering spaces of $\Lambda(2)$ and $S^{1}$. The covering space for the former is $C(2)=S^{2} \times \mathbf{R}$ and $\mathbf{R}$ for the circle. Indicating a lift by ${ }^{\wedge}$, given any $\psi \in \Lambda(2)$, its train is covered by $\hat{\mathcal{D}}(\phi)$. This can be viewed as the union of infinitely many adjacent sideways hourglasses. Each vertical slice is a disc and the fiber $S^{2}$ is obtained by identifying the entire boundary to a single point. Arnol'd shows that $\hat{\mathcal{D}}(\phi)$ divides $C(2)$ into infinitely many components. Each component is assigned a value of the Maslov Index which differs by +1 upon moving to an adjacent component. More precisely, the Maslov Index can be defined for a given path in $C(2)$. Consider the lift of the train of some $\beta \in \Lambda(2)$ in $C(2)$, denoted $\hat{\mathcal{D}}(\beta)$. 
Figure 1: The solid line depicts a path in $C(2)$ of Maslov Index 2. The dashed line depicts a path of Maslov Index 4.

Definition 3.6. The Maslov Index of the path starting at a point $\alpha$ and ending at $\beta^{\prime}, \alpha \notin \hat{\mathcal{D}}(\beta)$ is, by definition, either

(1) the index of intersection of this path with $\hat{\mathcal{D}}(\beta)$ if $\beta \neq \beta^{\prime}$, or

(2) $1+$ the index of intersection of this path with $\hat{\mathcal{D}}(\beta)$ if $\beta=\beta^{\prime}$.

The reason we need to discriminate between a path ending at $\beta$ and one not ending there is that the intersection of a path with the vertex is two-dimensional, whereas the intersection of a path with other parts of the train is one-dimensional. (See Figure 1)

Each hourglass is identical modulo a given period. The relation between $C(2)$ and $\mathbf{R}$ is given by the horizontal distance traveled in $C(2)$ projected onto $\mathbf{R}$, the covering space of $S^{1}$. Thus a path connecting the vertices of two adjacent hourglasses can be viewed in $\mathbf{R}$ as having traversed a distance of $2 \pi$. Alternatively, it can be viewed on $S^{1}$ as completing one full revolution on the circle.

Unfortunately, some information will be lost during the projection. For instance, should the right end point $\beta^{\prime}$ of a particular path lie at the vertex of a particular hourglass, then it will have the same projected distance onto $\mathbf{R}$ as a path whose right endpoint lies in the vertical disc containing that vertex. The Maslov Index of these two curves will in general differ by +1 . It appears that this difficulty will have to be addressed on a case by case basis.

Fix $\lambda$ and note that $\phi(\lambda,-1)$, the representative of $W_{-}^{u}$, is a point in $\Lambda(2)$. As the flow is applied to $\phi(\lambda, \tau)$, it defines a path in $\Lambda(2)$. This path can be lifted to $C(2)$ modulo a choice of left end point. The left end point will therefore define some point on $S^{1}$ modulo $2 \pi$.

Eigenvalues are created by a shooting argument in $C(2)$. Fix $\lambda_{1}<\lambda_{2}$ and obtain the parameterized flow on $C(2) \times[-1,+1] \times\left[\lambda_{1}, \lambda_{2}\right]$. Let $\hat{\Phi}\left(\left[\lambda_{1}, \lambda_{2}\right], \tau_{0}\right)=$ $\bigcup_{\left[\lambda_{1}, \lambda_{2}\right]} \hat{\Phi}(\lambda, \tau)$. Suppose that $\omega\left(\hat{\phi}\left(\lambda_{1}, \tau_{0}\right)\right)$ lies inside a particular component. If $\omega\left(\hat{\phi}\left(\lambda_{2}, \tau_{0}\right)\right)$ lies outside of this component, then by the fact that $\omega\left(\hat{\Phi}\left(\left[\lambda_{1}, \lambda_{2}\right], \tau_{0}\right)\right)$ is connected, there must be a $\lambda_{0} \in\left[\lambda_{1}, \lambda_{2}\right]$ for which $\omega\left(\hat{\phi}\left(\lambda_{0}, \tau_{0}\right)\right)$ intersects 
$\hat{\mathcal{D}}\left(\sigma\left(Z^{+1}\right)\right)$. A similar result is established in Jones [27]. Because of the monotonicity in $\lambda$, we will be able to get an exact count of eigenvalues in the interval $\left[\lambda_{1}, \lambda_{2}\right]$.

Denote by $S_{+}(\lambda)$ the stable subspace at $+\infty$. Also by $U_{ \pm}(\lambda)$ denote the unstable subspaces at $\pm \infty$. These subspaces can easily be characterized as elements of $\Lambda(2)$ using the stable and unstable eigenvectors at $\pm \infty$. Choose continuous lifts $\hat{U}_{ \pm}, \hat{S}_{+}, \hat{\mathcal{D}}\left(S_{+}(\lambda)\right)$. By $I(\lambda)$ denote the Maslov Index of the path in $C(2)$ with left endpoint $\hat{U}_{-}$and right endpoint $\omega\left(\hat{\phi}\left(\lambda, \tau_{0}\right)\right)$.

Lemma 3.7. If $\lambda_{1}<\lambda_{2}$, then the number of eigenvalues $\lambda \in\left[\lambda_{1}, \lambda_{2}\right)$ equals $I\left(\lambda_{1}\right)-I\left(\lambda_{2}\right)$.

Proof. First it is important to note that in projective or Grassmannian space the unstable subspace of a saddle point becomes a stable critical point and the stable subspace becomes an unstable critical point! In this case $\hat{U}_{+}(\lambda)$ is a stable critical point and $\hat{S}_{+}(\lambda)$ is a repelling critical point for the flow in $C(2)$. In $C(2)$, $\hat{U}_{+}(\lambda)$ can be visualized as lying in the interior of the disc that forms the left face of any hourglass. This choice is arbitrary modulo $2 \pi$. The critical point $\hat{S}_{+}(\lambda)$ can be visualized as the vertex of an hourglass, and $\hat{\mathcal{D}}\left(\hat{S}_{+}(\lambda)\right)$ is the boundary of the hourglass minus the right and left faces. Similarly $\hat{U}_{-}(\lambda)$ lies in $C(2)$. We fix a particular lift $\hat{U}_{-}(\lambda)$.

There are three possibilities for $\omega\left(\hat{\phi}\left(\lambda, \tau_{0}\right)\right)$ :

(1) $\omega\left(\hat{\phi}\left(\lambda, \tau_{0}\right)\right) \cap \hat{U}_{+} \neq \varnothing$, or

(2) $\omega\left(\hat{\phi}\left(\lambda, \tau_{0}\right)\right) \cap \hat{S}_{+} \neq \varnothing$, or

(3) $\omega\left(\hat{\phi}\left(\lambda, \tau_{0}\right)\right) \cap \hat{\mathcal{D}}\left(\hat{S}_{+}(\lambda)\right) \neq \varnothing$ and $\omega\left(\hat{\phi}\left(\lambda, \tau_{0}\right)\right) \cap \hat{S}_{+}=\varnothing$.

Depending on $\lambda$, any one of these three intersections can occur in one of infinitely many components. Independent of the component in which the intersection occurs, the Maslov Index of the path in question is well defined and computable.

In order to actually create an eigenvalue, either condition (2) or (3) would need to be satisfied. However, if, say, for $\lambda_{1}$ and $\lambda_{2}$, condition (1) is satisfied such that $I\left(\lambda_{1}\right)=a, I\left(\lambda_{2}\right)=b$ and $a>b$, then $\omega\left(\hat{\phi}\left(\lambda_{1}, \tau_{0}\right)\right)$ and $\omega\left(\hat{\phi}\left(\lambda_{2}, \tau_{0}\right)\right)$ lie in different components of $C(2)$. Therefore, by connectedness of $\omega\left(\Phi\left(\left[\lambda_{1}, \lambda_{2}\right], \tau_{0}\right)\right.$, the path connecting $\omega\left(\hat{\phi}\left(\lambda_{2}, \tau_{0}\right)\right)$ and $\omega\left(\hat{\phi}\left(\lambda_{1}, \tau_{0}\right)\right)$ must have intersected the train of $\bigcup_{\left[\lambda_{1}, \lambda_{2}\right]} \hat{S}_{+}(\lambda)$ at least $a-b$ times. We need to establish that $\omega\left(\hat{\Phi}\left(\left[\lambda_{1}, \lambda_{2}\right], \tau_{0}\right)\right.$ intersects the train of $\bigcup_{\left[\lambda_{1}, \lambda_{2}\right]} \hat{S}_{+}(\lambda)$ exactly $a-b$ times. For the sake of argument, it suffices to show this for $b=a-1$. Thus, we claim that the number of eigenvalues in $\left[\lambda_{1}, \lambda_{2}\right]$ is exactly 1 . Suppose $\omega\left(\hat{\Phi}\left(\left[\lambda_{1}, \lambda_{2}\right], \tau_{0}\right)\right.$ intersects the train of $\bigcup_{\left[\lambda_{1}, \lambda_{2}\right]} \hat{S}_{+}(\lambda)$ more than once. This implies, by connectedness of $\omega$-limit sets, that there exist at least two eigenvalues $\lambda_{3}$ and $\lambda_{4}$, with $\lambda_{1}<\lambda_{3}<\lambda_{4}<\lambda_{2}$, such that $\omega\left(\hat{\phi}\left(\lambda_{3}, \tau_{0}\right)\right) \cap \hat{\mathcal{D}}\left(\hat{S}_{+}\left(\lambda_{3}\right)\right) \neq \varnothing$ and $\omega\left(\hat{\phi}\left(\lambda_{4}, \tau_{0}\right)\right) \cap \hat{\mathcal{D}}\left(\hat{S}_{+}\left(\lambda_{4}\right)\right) \neq \varnothing$. 
Again, by the connectedness of $\omega$-limit sets, the path connecting $\omega\left(\hat{\phi}\left(\lambda_{2}, \tau_{0}\right)\right)$ and $\omega\left(\hat{\phi}\left(\lambda_{3}, \tau_{0}\right)\right)$ intersects $\hat{\mathcal{D}}\left(\bigcup_{\left[\lambda_{2}, \lambda_{3}\right]} \hat{S}_{+}(\lambda)\right)$ just once at $\lambda=\lambda_{3}$. However, the path connecting $\omega\left(\hat{\phi}\left(\lambda_{2}, \tau_{0}\right)\right)$ and $\omega\left(\hat{\phi}\left(\lambda_{4}, \tau_{0}\right)\right)$ will intersect $\hat{\mathcal{D}}\left(\bigcup_{\left[\lambda_{2}, \lambda_{4}\right]} \hat{S}_{+}(\lambda)\right)$ twice. Since $I\left(\lambda_{2}\right)=a-1$, this implies that $I\left(\lambda_{4}\right)=a+1$. Thus, $I\left(\lambda_{4}\right)>I\left(\lambda_{1}\right)$. But $\lambda_{4}>\lambda_{1}$, so by Lemma 3.3 this contradicts the fact that $\kappa$, and therefore $I(\lambda)$, is monotone decreasing in $\lambda$. It is easy to extend this argument to the more general case as needed. Thus, we conclude that the path connecting $\omega\left(\hat{\phi}\left(\lambda_{2}, \tau_{0}\right)\right)$ and $\omega\left(\hat{\phi}\left(\lambda_{1}, \tau_{0}\right)\right)$ intersects $\hat{\mathcal{D}}\left(\bigcup_{\left[\lambda_{1}, \lambda_{2}\right]} \hat{S}_{+}(\lambda)\right)$ exactly $a-b$ times. Similar reasoning holds for the other possible cases of $\lambda_{1}$ and $\lambda_{2}$. The lemma holds on the closed interval $\left[\lambda_{1}, \lambda_{2}\right]$ if $\lambda_{2}$ is not an eigenvalue. If $\lambda_{2}$ is an eigenvalue, then the number of eigenvalues in the closed interval $\left[\lambda_{1}, \lambda_{2}\right]$ is $1+I\left(\lambda_{1}\right)-I\left(\lambda_{2}\right)$.

An immediate consequence of Lemmas 3.4 and 3.7 is the following corollary.

Corollary 3.8. The number of eigenvalues $\lambda \in\left[\lambda_{1},+\infty\right)$ equals $I\left(\lambda_{1}\right)$.

Proof. We establish that if $\lambda_{2} \gg 1$, then $I\left(\lambda_{2}\right)=0$. It was shown in Lemma 3.4 that as $\lambda_{2} \rightarrow \infty,(\partial / \partial \kappa)(\lambda, \xi) \rightarrow 0$. In fact, as $\lambda_{2} \rightarrow \infty, \hat{U}_{-}\left(\lambda_{2}\right) \rightarrow$ $\hat{U}_{+}\left(\lambda_{2}\right)$. Therefore, $\hat{\phi}\left(\lambda_{2}, \tau\right)$ stays near to $\hat{U}_{+}\left(\lambda_{2}\right)$ for all $\tau$. Thus, it stays in the same component of $C(2)$. Call this component $\mathcal{A}$. Therefore, if $\lambda_{2} \gg 1$, $\omega\left(\hat{\phi}\left(\lambda_{2}, \tau_{0}\right)\right) \cap \hat{U}_{+} \subset \mathcal{A}$ and is non-empty. Thus $I\left(\lambda_{2}\right)=0$. This is to say that there are no eigenvalues for $\lambda$ large.

3.3. Analysis for the singular in-phase front and Keener's model. We are now in a position to apply this general theory to the specific equations under consideration in this paper. We first conclude the proof of Theorem 2.6, and we then present a detailed application of the theory to Keener's model.

Proof of Theorem 2.6. It follows from the transversality hypothesis in (H4) and from the work of Alexander and Jones [2] that 0 is a simple eigenvalue of the origin. Also, in (H8) we assumed that the travelling wave has Maslov Index 1. The Maslov Index is assigned to the wave at the value $\lambda=0$. Thus Corollary 3.8 implies that there exists only one eigenvalue of the linear operator $L_{F}$ in the right half-plane. This eigenvalue must then be the simple one at the origin, thus proving Theorem 2.6.

Recall that in Keener's model the non-linearity $f(U)$ was given by

$$
f(U)=\left(\begin{array}{c}
g\left(u_{1}\right)+d\left(u_{2}-u_{1}\right) \\
g\left(u_{2}\right)+d\left(u_{1}-u_{2}\right)
\end{array}\right),
$$


where $g(u)=u(1-u)(u-a)$ and $d$ is the coupling coefficient. When $d>0$, the coupling is excitatory, and when $d<0$, it is inhibitory. Bose [7] shows that for $d$ sufficiently small, the symmetric in-phase front travelling wave solution can be constructed as the transverse intersection of relevant manifolds. In the following, we adopt the notation of the previous section and apply it to the equations and solution at hand. Thus, denote the in-phase solution $U_{F}(\xi)$ with associated linear operator $L_{F}$. The eigenvalue equation $\left(L_{F}-\lambda I\right) P=0$ is given by

$$
\begin{aligned}
& p_{1}^{\prime}=q_{1} \\
& p_{2}^{\prime}=q_{2} \\
& q_{1}^{\prime}=\vartheta^{*} q_{1}+\left(\lambda-\frac{\partial g}{\partial u_{1}}\right) p_{1}-d\left(p_{2}-p_{1}\right) \\
& q_{2}^{\prime}=\vartheta^{*} q_{2}+\left(\lambda-\frac{\partial g}{\partial u_{2}}\right) p_{2}-d\left(p_{1}-p_{2}\right) .
\end{aligned}
$$

Note that, at $\lambda=0,(28)$ is exactly the equation of variations for the front. Evolution equations for the six 2-forms associated with (28) are

$$
\begin{aligned}
P_{12}^{\prime} & =P_{14}-P_{23} \\
P_{13}^{\prime} & =\vartheta^{*} P_{13}-d P_{12} \\
P_{24}^{\prime} & =\vartheta^{*} P_{24}+d P_{12} \\
P_{14}^{\prime}= & \vartheta^{*} P_{14}-\frac{\partial g}{\partial u_{2}} P_{12}+d P_{12}+P_{34}+\lambda P_{12} \\
P_{23}^{\prime}= & \vartheta^{*} P_{23}+\frac{\partial g}{\partial u_{1}} P_{12}-d P_{12}-P_{34}-\lambda P_{12} \\
P_{34}^{\prime}= & 2 \vartheta^{*} P_{34}+\left(d-\frac{\partial g}{\partial u_{1}}\right) P_{14}-\left(d-\frac{\partial g}{\partial u_{2}}\right) P_{23} \\
& +d\left(P_{13}-P_{24}\right)+\lambda\left(P_{14}-P_{23}\right) .
\end{aligned}
$$

We will now consider the dependence on the parameter $d$ as well as $\lambda$ by noting $\kappa=\kappa(\lambda, \xi, d), U_{ \pm}=U_{ \pm}(\lambda, d)$ and $S_{+}=S_{+}(\lambda, d)$. There are two more pieces of information which are specific to the singular front of Keener's model which are contained in Lemmas 3.9 and 3.10. For $d=0, \lambda=0, W_{-}^{u} \cap W_{+}^{s}$ is two-dimensional since these subspaces coincide. Moreover, the following is true:

Lemma 3.9. For the in-phase singular front solution, when $d=0$, $I(0)=2$. 
Proof. For $d=0$, the systems uncouple and we recover two copies of the FitzHugh-Nagumo front, each of which is known to be stable. By Corollary 3.8, $I(0) \leq 2$. Since $\operatorname{dim}\left(W_{-}^{u} \cap W_{+}^{s}\right)=2, \omega\left(\hat{\phi}\left(0, \tau_{0}\right)\right) \cap \hat{S}_{+}(0,0) \neq 0$, i.e. $\omega\left(\hat{\phi}\left(0, \tau_{0}\right)\right)$ must be the vertex of $\hat{\mathcal{D}}\left(\hat{S}_{+}(, 0,0)\right)$. Thus, it follows that $I(0)=2$.

As in Lemma 3.3, denote the dependence of $\kappa$ on $d$ by a subscript.

Lemma 3.10. For fixed $\lambda$ and for each $\xi$, if $d_{1}<d_{2}$ then $\kappa_{d_{1}}(\xi)>\kappa_{d_{2}}(\xi)$.

We omit the proof of Lemma 3.10, since it is nearly identical to that of Lemma 3.3.

Theorem 3.11. For $d>0$ and sufficiently small, there exist no eigenvalues of $L_{F}$ in the open right half-plane. For $d<0$ and sufficiently small, there exists one eigenvalue in the open right half-plane.

Notice that the sign of the coupling coefficient $d$ is the determining factor for stability.

Proof. For $d=0,0$ is an eigenvalue of multiplicity 2. Using the transversality result of Bose [7], for $d$ sufficiently small, 0 is a simple eigenvalue of $L_{F}$. We need to determine the behavior of the second eigenvalue as $d$ is perturbed from 0 .

The theorem will be proved by applying Corollary 3.8 with $\lambda_{1}=0$ and $d$ sufficiently small. For $d=0$, by Lemma $3.9, I(0)=2$. Now choose $d_{1}<0<d_{2}$ sufficiently small. Using the stable eigenvectors, it can be shown that if $d_{2}-d_{1}$ is sufficiently small, then $\hat{S}_{+}\left(0, d_{1}\right)<\hat{S}_{+}\left(0, d_{2}\right)$. By this we mean that the projection of $\hat{S}_{+}\left(0, d_{1}\right)$ onto the real line lies to the left of the projection of $\hat{S}_{+}\left(0, d_{2}\right)$. Thus, a component of the train of $\hat{S}_{+}\left(0, d_{1}\right)$ lies to the left of an analogous component of the train of $\hat{S}_{+}\left(0, d_{2}\right)$. Figure 2 shows the relative positions of one component of each train and the relevant paths for $d_{1}$ and $d_{2}$. Now, by Lemma 3.10 , since $\kappa$ is monotone decreasing in $d$, for $d_{2}>0, \omega\left(\hat{\phi}\left(0, \tau_{0}\right)\right)$ gets pushed to the left into the same component as $\hat{U}_{-}\left(0, d_{2}\right)$. Since 0 is an eigenvalue, $\lambda_{1}=0$ satisfies condition (3) in the proof of Lemma 3.7. That is $\omega\left(\hat{\phi}\left(0, \tau_{0}\right)\right) \cap \hat{\mathcal{D}}\left(\hat{S}_{+}\left(0, d_{2}\right)\right) \neq \varnothing$. This is to say that $\operatorname{dim}\left(W_{-}^{u} \cap W_{+}^{s}\right)=1$. Therefore $I(0)=1$. For $d_{1}<0$ the opposite happens. Since $\kappa$ increases, $\omega\left(\hat{\phi}\left(0, \tau_{0}\right)\right)$ gets pushed to the right into a different component than $\hat{U}_{-}\left(0, d_{1}\right)$. Again 0 is an eigenvalue, so condition (3) is satisfied. However, since $\omega\left(\hat{\phi}\left(0, \tau_{0}\right)\right)$ lies in the component to the right of the one in which $\hat{U}_{-}\left(0, d_{1}\right)$ lies, $I(0)=2$.

Thus, for $d>0, I(0)-I(\infty)=1$, which corresponds to the eigenvalue at the origin. Therefore, there exist no eigenvalues in the right half-plane and the in-phase front solution is stable. For $d<0, I(0)-I(\infty)=2$, one of which corresponds to the zero eigenvalue which is simple. Therefore, the other must lie in the right half-plane. Thus, in this case the in-phase front solution is unstable. 
FIGURE 2: The solid line depicts the path of a trajectory associated with $d_{2}>0$ and has Maslov Index 1 . The dashed line depicts a trajectory associated with $d_{1}<0$ and has Maslov Index 2. 
The fact that the direction of transversality of the in-phase solution changes as $d$ passes through 0 (as shown in [7]) is the cause for this change in stability of the solution.

4. Stability for the full $\varepsilon \neq 0$ wave. In this section we conclude the proof of stability for the full $\varepsilon \neq 0$ system. In order to finish the proof, two additional pieces of information are required. First, for the linear operator $L$, it must be shown that 0 is a simple eigenvalue. Second, the other eigenvalue of $L$ inside of $K$ must be shown to lie in the left half-plane. These two requirements are intertwined and obtained by evaluating $D^{\prime}(0)$. This will be achieved using the Exchange Lemma to exploit information obtained from the singular solutions.

We will show that 0 is simple by proving that $D^{\prime}(0)>0$. That 0 is simple forces the second eigenvalue to be real, since eigenvalues come in complex conjugate pairs. Using our orientation conventions, it can be shown that $D(\lambda \gg 1)>0$. Since $D(\lambda)$ is analytic in $\lambda$, by establishing that $D^{\prime}(0)>0$, we will also have shown that the other eigenvalue lies in the left half-plane.

We will establish that $D^{\prime}(0)>0$ as a direct consequence of the Exchange Lemma. The idea is the following. As noted earlier, the eigenvalue equations at $\lambda=0$ are exactly the equations of variation, which are the equations that govern the evolution of tangent vectors under the flow. Thus, at $\lambda=0$, information about $D(\lambda)$ can be obtained from tangent vectors. The Exchange Lemma allows us to obtain the $C^{1}$ closeness of certain tangent planes of the $\varepsilon=0$ and $\varepsilon \neq 0$ systems. This will, in turn, enable us to prove the closeness of certain objects associated with $D^{\prime}(0)$ and with the $\varepsilon=0$ reduced systems that are determined by these tangent planes. Obtaining the sign of $D^{\prime}(0)$ amounts to checking the orientation of a certain tangent hyperplane after it has evolved under the flow to a point near manifold $(\mathrm{R})$ close to the singular back. There is no easy way of directly determining this orientation. However, the Exchange Lemma picks out a tangent hyperplane associated with the singular back, whose orientation is known, which is $O(\varepsilon)$ close to the unknown one.

At this time, we also note that using the Exchange Lemma is crucial. It can happen that although both jumps are stable relative to their reduced systems, together they may produce an unstable wave. By using the Exchange Lemma, we guarantee that information about the slow flow is utilized in determining stability of the full solution.

Theorem 4.1. If $\varepsilon$ is sufficiently small, the derivative $D^{\prime}(0)>0$. 
Proof. Appending $\vartheta^{\prime}=0$ to the travelling wave equations (4), we obtain

$$
\begin{aligned}
u_{1}^{\prime} & =w_{1} \\
u_{2}^{\prime} & =w_{2} \\
w_{1}^{\prime} & =\vartheta w_{1}-f_{1}\left(u_{1}, u_{2}\right)+v_{1} \\
w_{2}^{\prime} & =\vartheta w_{2}-f_{2}\left(u_{1}, u_{2}\right)+v_{2} \\
v_{1}^{\prime} & =\frac{\varepsilon}{\vartheta}\left(u_{1}-\gamma v_{1}\right) \\
v_{1}^{\prime} & =\frac{\varepsilon}{\vartheta}\left(u_{2}-\gamma v_{2}\right) \\
\vartheta^{\prime} & =0 .
\end{aligned}
$$

The origin has a three-dimensional $W^{c u}(0)$ and a five-dimensional $W^{c s}(0)$. Let $\Gamma_{\varepsilon}$ be the homoclinic solution. Let $\Gamma^{+}\left(\Gamma^{-}\right)$be a component of $W^{c u}(0)\left(W^{c s}(0)\right)$ which contains $\Gamma_{\varepsilon}$. The derivative of the Evans function can be related to the manner in which $W^{u}(0)$ crosses $W^{s}(0)$ as $\vartheta$ varies. To this end, let $X_{1}^{ \pm}(\lambda, \xi)=$ $\mathcal{V}$, where $\mathcal{V}$ is the vector field of (30). Suppressing dependence on $(\lambda, \xi)$ for notational convenience, as in [2], we can establish

$$
\begin{aligned}
D^{\prime}(0)=e^{-\int_{0}^{\xi} T R A(\lambda, \xi) d \xi}\left(\left.\frac{d}{d \vartheta}\left(\Gamma_{+}-\Gamma_{-}\right)\right|_{\vartheta=\vartheta(\varepsilon)}\right. & \\
& \left.\wedge X_{2}^{+} \wedge \mathcal{V} \wedge X_{2}^{-} \wedge X_{3}^{-} \wedge X_{4}^{-}\right) .
\end{aligned}
$$

We must evaluate $D^{\prime}(0)$ somewhere near the singular back in order to account for the slow flow. The following lemma allows us to understand the passage of $\Gamma_{\varepsilon}$ near to manifold (E).

Lemma 4.2. (Jones, Kopell [28]) Let $B^{1}$ be a box around manifold $(E)$ in which we have Fenichel coordinates. Let $q_{1}$ be a point on the singular orbit on the entrance $|b|=\Delta$ to $B^{1}$ and let $\bar{q}_{1}$ be the point on $\{|a|=\Delta\}$ at which the singular orbit exits $B^{1}$. Then, for any point $\bar{p}_{1} \in\left\{|a|=\Delta, b=0, y_{i}=0, i>1\right\}$ sufficiently close to $\bar{q}_{1}$, there is a point $q$ near $q_{1}$ such that the trajectory through $q$ reaches $\{|a|=\Delta\}$ at a point $\bar{q}$ having the same $a$ and $y_{1}$ coordinates as $\bar{p}_{1}$ and nearby $b, y_{i}$ coordinates, $i>1$. The time from $q$ to $\bar{q}$ is $O(1 / \varepsilon)$.

Choose $B^{1}$ such that $w_{1}$ and $w_{2}$ are $O(\delta)$ inside $B^{1}$, where $\delta$ is the length of the shortest side of $B^{1}$ and is fixed small independent of $\varepsilon$. Let $T_{0}^{\varepsilon}$ be the value of $\xi$ at which $\Gamma_{\varepsilon}$ enters $B^{1}$ and $T_{1}^{\varepsilon}$ the value at which it leaves. Define $T_{0}$ and $T_{1}$ similarly for the singular orbit. Let $q_{\varepsilon} \in \Gamma_{\varepsilon}$ be a point somewhere near manifold $(\mathrm{R})$ close to the singular back. Furthermore, let $\Gamma_{\varepsilon}$ be parameterized such that $\xi=\tau_{\varepsilon}$ at $q_{\varepsilon}$. Near $q_{\varepsilon}, W^{c u}(0)$ and $W^{c s}(0)$ 
can locally be written as the graphs of functions. Let $W^{c u}(0)$ be given by $\left(u_{1}, u_{2}, m_{\varepsilon}^{+}\left(u_{1}, u_{2}, \vartheta\right), n_{\varepsilon}^{+}\left(u_{1}, u_{2}, \vartheta\right), a_{\varepsilon}^{+}\left(u_{1}, u_{2}, \vartheta\right), b_{\varepsilon}^{+}\left(u_{1}, u_{2}, \vartheta\right), \vartheta\right)$, and $W^{c s}(0)$ by $\left(u_{1}, u_{2}, m_{\varepsilon}^{-}\left(u_{1}, u_{2}, v_{1}, v_{2}, \vartheta\right), n_{\varepsilon}^{-}\left(u_{1}, u_{2}, v_{1}, v_{2}, \vartheta\right), v_{1}, v_{2}, \vartheta\right)$. Tangent vectors to the various manifolds can be found by taking derivatives of these graphs with respect to different variables. Let

$$
\begin{aligned}
V_{u}^{\vartheta} & =\left(0,0, \frac{\partial}{\partial \vartheta} m_{\varepsilon}^{+}\left(q_{\varepsilon}\right), \frac{\partial}{\partial \vartheta} n_{\varepsilon}^{+}\left(q_{\varepsilon}\right), \frac{\partial}{\partial \vartheta} a_{\varepsilon}^{+}\left(q_{\varepsilon}\right), \frac{\partial}{\partial \vartheta} b_{\varepsilon}^{+}\left(q_{\varepsilon}\right), 1\right) \\
V_{u}^{1} & =\left(1,0, \frac{\partial}{\partial u_{1}} m_{\varepsilon}^{+}\left(q_{\varepsilon}\right), \frac{\partial}{\partial u_{1}} n_{\varepsilon}^{+}\left(q_{\varepsilon}\right), \frac{\partial}{\partial u_{1}} a_{\varepsilon}^{+}\left(q_{\varepsilon}\right), \frac{\partial}{\partial u_{1}} b_{\varepsilon}^{+}\left(q_{\varepsilon}\right), 0\right), \\
V_{s}^{\vartheta} & =\left(0,0, \frac{\partial}{\partial \vartheta} m_{\varepsilon}^{-}\left(q_{\varepsilon}\right), \frac{\partial}{\partial \vartheta} n_{\varepsilon}^{-}\left(q_{\varepsilon}\right), 0,0,1\right), \\
V_{s}^{1} & =\left(1,0, \frac{\partial}{\partial u_{1}} m_{\varepsilon}^{-}\left(q_{\varepsilon}\right), \frac{\partial}{\partial u_{1}} n_{\varepsilon}^{-}\left(q_{\varepsilon}\right), 0,0,0\right), \\
V_{s}^{2} & =\left(0,0, \frac{\partial}{\partial v_{1}} m_{\varepsilon}^{-}\left(q_{\varepsilon}\right), \frac{\partial}{\partial v_{1}} n_{\varepsilon}^{-}\left(q_{\varepsilon}\right), 1,0,0\right), \\
V_{s}^{3} & =\left(0,0, \frac{\partial}{\partial v_{2}} m_{\varepsilon}^{-}\left(q_{\varepsilon}\right), \frac{\partial}{\partial v_{2}} n_{\varepsilon}^{-}\left(q_{\varepsilon}\right), 0,1,0\right),
\end{aligned}
$$

be such that

$$
\begin{aligned}
& X_{2}^{+}\left(0, \tau_{\varepsilon}\right)=V_{u}^{1} \quad, \quad X_{3}^{-}\left(0, \tau_{\varepsilon}\right)=V_{s}^{2} \\
& \frac{d}{d \vartheta} \Gamma_{+}\left(\tau_{\varepsilon}\right)=V_{u}^{\vartheta} \quad, \quad \frac{d}{d \vartheta} \Gamma_{-}\left(\tau_{\varepsilon}\right)=V_{s}^{\vartheta} \\
& X_{2}^{-}\left(0, \tau_{\varepsilon}\right)=V_{s}^{1} \quad, \quad X_{4}^{-}\left(0, \tau_{\varepsilon}\right)=V_{s}^{3} \text {. }
\end{aligned}
$$

Note that, at $\xi=\tau_{\varepsilon}$,

$$
\operatorname{sgn} D^{\prime}(0)=\operatorname{sgn}\left(V_{u}^{\vartheta}-V_{s}^{\vartheta}\right) \wedge V_{u}^{1} \wedge \mathcal{V} \wedge V_{s}^{1} \wedge V_{s}^{2} \wedge V_{s}^{3}
$$

Below, we use differential 3-forms to make certain calculations. These forms are found in the same way as in previous sections by using the product rule together with the equation of variations of (30). Let $\Pi_{\varepsilon}$ be the hyperplane spanned by $\left(V_{u}^{\vartheta}-V_{s}^{\vartheta}\right),\left(V_{u}^{1}-V_{s}^{1}\right)$ and $\mathcal{V}$. Next observe that $\left(V_{u}^{\vartheta}-V_{s}^{\vartheta}\right) \wedge V_{u}^{1} \wedge \mathcal{V} \wedge V_{s}^{1} \wedge V_{s}^{2} \wedge V_{s}^{3}=$ $-\left(P_{u_{2} w_{1} w_{2}}^{\varepsilon}\left[\Pi_{\varepsilon}\right]\left(\tau_{\varepsilon}\right)\right)+O(\varepsilon)$. That the error is only $O(\varepsilon)$ will be justified by the estimates below. Therefore

$$
\operatorname{sgn} D^{\prime}(0)=-\operatorname{sgn}\left(P_{u_{2} w_{1} w_{2}}^{\varepsilon}\left[\Pi_{\varepsilon}\right]\left(\tau_{\varepsilon}\right)\right)
$$

As a result of $(33)$, we need to find the sign of $P_{u_{2} w_{1} w_{2}}^{\varepsilon}\left[\Pi_{\varepsilon}\right]\left(\tau_{\varepsilon}\right)$. 
Lemma 4.3. The 3-form $P_{u_{2} w_{1} w_{2}}^{\varepsilon}\left[\Pi_{\varepsilon}\right]\left(\tau_{\varepsilon}\right)<0$.

Proof. In the following, any term with the super/subscript ' $f$ ' or ' $b$ ' refers to the object associated with the $\varepsilon=0$ singular front or back, respectively.

For the singular front, at $\xi=T_{0}$, let the three-dimensional tangent hyperplane, $H_{f}$, be spanned by

$$
\begin{aligned}
& \left(0,0, \frac{\partial}{\partial \vartheta} \Delta g_{f}\left(q_{1}\right), \frac{\partial}{\partial \vartheta} \Delta h_{f}\left(q_{1}\right), 0,0,1\right) \\
& \left(1,0, \frac{\partial}{\partial u_{1}} \Delta g_{f}\left(q_{1}\right), \frac{\partial}{\partial u_{1}} \Delta h_{f}\left(q_{1}\right), 0,0,0\right) \\
& \left(w_{1}, w_{2}, w_{1}^{\prime}, w_{2}^{\prime}, 0,0,0\right),
\end{aligned}
$$

where $W_{F}^{c u}(0), W_{F}^{c s}\left(U_{+}(0)\right)$ are locally the graphs of $\left(u_{1}, u_{2}, g_{f}^{+}\left(u_{1}, u_{2}, \vartheta\right)\right.$, $\left.h_{f}^{+}\left(u_{1}, u_{2}, \vartheta\right), 0,0, \vartheta\right)$ and $\left(u_{1}, u_{2}, g_{f}^{-}\left(u_{1}, u_{2}, \vartheta\right), h_{f}^{-}\left(u_{1}, u_{2}, \vartheta\right), 0,0, \vartheta\right)$ respectively, and $\Delta g_{f}=g_{f}^{+}-g_{f}^{-}$etc. For $\varepsilon \neq 0$ and sufficiently small, these graphs can be smoothly perturbed such that near $\xi=T_{0}^{\varepsilon}$, for fixed $\varepsilon, W^{c u}(0)$ is the graph of $\left(u_{1}, u_{2}, g_{\varepsilon}^{+}\left(u_{1}, u_{2}, \vartheta\right), h_{\varepsilon}^{+}\left(u_{1}, u_{2}, \vartheta\right), r_{\varepsilon}^{+}\left(u_{1}, u_{2}, \vartheta\right), s_{\varepsilon}^{+}\left(u_{1}, u_{2}, \vartheta\right), \vartheta\right)$ and $W^{s}\left(E_{\varepsilon}\right)$ is the graph of $\left(u_{1}, u_{2}, g_{\varepsilon}^{-}\left(u_{1}, u_{2}, \vartheta\right), h_{\varepsilon}^{-}\left(u_{1}, u_{2}, \vartheta\right), r_{\varepsilon}^{-}\left(u_{1}, u_{2}, \vartheta\right), s_{\varepsilon}^{-}\left(u_{1}, u_{2}, \vartheta\right), \vartheta\right)$. As a result of the Exchange Lemma, $W^{c u}(0)$ transversely intersects $W^{s}\left(E_{\varepsilon}\right)$. Now let $H_{\varepsilon}^{0}$ be the three-dimensional hyperplane spanned by

$$
\begin{aligned}
& \left(0,0, \frac{\partial}{\partial \vartheta} \Delta g_{\varepsilon}(q), \frac{\partial}{\partial \vartheta} \Delta h_{\varepsilon}(q), \frac{\partial}{\partial \vartheta} \Delta r_{\varepsilon}(q), \frac{\partial}{\partial \vartheta} \Delta s_{\varepsilon}(q), 1\right) \\
& \left(1,0, \frac{\partial}{\partial u_{1}} \Delta g_{\varepsilon}(q), \frac{\partial}{\partial u_{1}} \Delta h_{\varepsilon}(q), \frac{\partial}{\partial u_{1}} \Delta r_{\varepsilon}(q), \frac{\partial}{\partial u_{1}} \Delta s_{\varepsilon}(q), 0\right) \\
& \left(w_{1}, w_{2}, w_{1}^{\prime}, w_{2}^{\prime}, v_{1}^{\prime}, v_{2}^{\prime}, 0\right) .
\end{aligned}
$$

Notice that by construction $(\partial / \partial \vartheta) \Delta g_{\varepsilon}=(\partial / \partial \vartheta) \Delta g_{f}+O(\varepsilon)$ and similarly for all other appropriate terms. Thus, sgn $P_{u_{2} w_{1} w_{2}}^{\varepsilon}\left[H_{\varepsilon}^{0}\right]\left(T_{0}^{\varepsilon}\right)=\operatorname{sgn} P_{u_{2} w_{1} w_{2}}^{f}\left[H_{f}\right]\left(T_{0}\right)$, provided that the latter is not $O(\varepsilon)$. We later show that it is not. Moreover, notice that $H_{\varepsilon}^{0}$ is constructed using the vector field, and vectors in the $\vartheta$ and $u_{1}$ directions, which is consistent with $\left(V_{u}^{\vartheta}-V_{s}^{\vartheta}\right) \wedge\left(V_{u}^{1}-V_{s}^{1}\right) \wedge \mathcal{V}$ at $\xi=\tau_{\varepsilon}$.

Denote by $H_{\varepsilon}^{1}$ the evolution of the hyperplane $H_{\varepsilon}^{0}$ under the flow to the time $T_{1}^{\varepsilon}$. The Exchange Lemma implies that $P_{u_{2} w_{1} w_{2}}^{\varepsilon}\left[H_{\varepsilon}^{1}\right]\left(T_{1}^{\varepsilon}\right)$ is $O(\varepsilon)$ close to $P_{u_{2} w_{1} w_{2}}^{b}\left[H_{b}\right]\left(T_{1}\right)$ up to normalization and sign for an appropriate hyperplane $H_{b}$ which is determined by the transversality of the back travelling wave. That is, for some $k_{1}$,

$$
P_{u_{2} w_{1} w_{2}}^{\varepsilon}\left[H_{\varepsilon}^{1}\right]\left(T_{1}^{\varepsilon}\right)=k_{1} P_{u_{2} w_{1} w_{2}}^{b}\left[H_{b}\right]\left(T_{1}\right)+O(\varepsilon) .
$$


The sign, and thus orientation, of $P_{u_{2} w_{1} w_{2}}^{b}\left[H_{b}\right]\left(T_{1}\right)$ is determined by the $\varepsilon=0$ transversality result of the back. Without loss of generality, we take

$$
P_{u_{2} w_{1} w_{2}}^{b}\left[H_{b}\right]\left(T_{1}\right)>0 .
$$

Since $\operatorname{sgn} P_{u_{2} w_{1} w_{2}}^{\varepsilon}\left[H_{\varepsilon}^{0}\right]\left(T_{0}^{\varepsilon}\right)=\operatorname{sgn} P_{u_{2} w_{1} w_{2}}^{f}\left[H_{f}\right]\left(T_{0}\right)$, the sign of $k_{1}$ is determined by the behavior of $P_{u_{2} w_{1} w_{2}}^{\varepsilon}$ as it passes by manifold (E). As we show below, $P_{u_{2} w_{1} w_{2}}^{\varepsilon}$ does not change sign as it passes by manifold (E). An important observation is then that $P_{u_{2} w_{1} w_{2}}^{b}$ maintains its sign as it tracks along the singular back. Using Gronwall's Inequality, $P_{u_{2} w_{1} w_{2}}^{\varepsilon}$ also will not change sign along the back, so we will be able to obtain the sign of $P_{u_{2} w_{1} w_{2}}^{\varepsilon}\left[\Pi_{\varepsilon}\right]\left(\tau_{\varepsilon}\right)$.

For convenience, suppress the dependence on the hyperplanes in question. We first determine the magnitude of $P_{u_{2} w_{1} w_{2}}^{f}\left(T_{0}\right)$. Note that $P_{u_{2} w_{1} w_{2}}^{f}\left(T_{0}\right) \neq 0$ by the transversality of the front.

$$
P_{u_{2} w_{1} w_{2}}^{f}\left(T_{0}\right)=w_{2}\left[\frac{\partial}{\partial \vartheta} \Delta g_{f} \frac{\partial}{\partial u_{1}} \Delta h_{f}-\frac{\partial}{\partial u_{1}} \Delta g_{f} \frac{\partial}{\partial \vartheta} \Delta h_{f}\right] .
$$

Using 2-forms and calculations similar to those in [7], it is not hard to show that $\left(\partial / \partial u_{1}\right) \Delta g_{f}=-\left(\partial / \partial u_{1}\right) \Delta h_{f}$ and both $(\partial / \partial \vartheta) \Delta g_{f}$ and $(\partial / \partial \vartheta) \Delta h_{f}$ are $O\left(e^{\vartheta T_{0}}\right)$ and positive. By comparison, the magnitudes of $\left(\partial / \partial u_{1}\right) \Delta g_{f}$ and $\left(\partial / \partial u_{1}\right) \Delta h_{f}$, which are independent of $\varepsilon$, can be taken without loss of generality to be at least $O(1)$.

The sign of $P_{u_{2} w_{1} w_{2}}^{f}\left(T_{0}\right)$ is determined by the transversality and stability of the front. Let $D_{F}(\lambda)=C \cdot Y_{1}^{+} \wedge Y_{2}^{+} \wedge Y_{1}^{-} \wedge Y_{2}^{-}$be the Evans function associated with the singular front. The constant $C$ is chosen by Abel's formula so as to make $D_{F}(\lambda)$ independent of $\xi$. Similar to what was done for the Evans function for the full system, choose the vectors $Y_{2}^{ \pm}$to agree with tangent vectors in the $u_{1}$ direction at the point $q_{1}$. Then the derivative $D_{F}^{\prime}(0)=$ $C(\partial / \partial \vartheta)\left(\Gamma_{f}^{+}-\Gamma_{f}^{-}\right) \wedge v_{f_{u}}^{1} \wedge \mathcal{V} \wedge v_{f_{s}}^{1}$ where $v_{f_{u}}^{1}$ and $v_{f_{s}}^{1}$ are the tangent vectors to $W_{F}^{c u}(0)$ and $W_{F}^{c s}\left(U_{+}(0)\right)$ mentioned above, and $\Gamma_{f}^{+}$and $\Gamma_{f}^{-}$are the smoothly extended components of the singular front in each of these manifolds. It then follows that $D_{F}^{\prime}(0)=-P_{u_{2} w_{1} w_{2}}^{f}\left(T_{0}\right)$. It can be shown that, by construction, $D_{F}(\lambda \gg 1)>0$. Since the front is stable, this implies $D_{F}^{\prime}(0)>0$, from which it follows that $P_{u_{2} w_{1} w_{2}}^{f}\left(T_{0}\right)<0$. Thus $P_{u_{2} w_{1} w_{2}}^{f}\left(T_{0}\right)=-c e^{\vartheta T_{0}}$ for $c>0$, and therefore $P_{u_{2} w_{1} w_{2}}^{\varepsilon}\left(T_{0}^{\varepsilon}\right)=O\left(e^{\vartheta T_{0}}\right)$ and is negative.

At $\xi=T_{0}, P_{u_{2} u_{1} w_{1}}^{f}\left(T_{0}\right)=-w_{2}(\partial / \partial \vartheta) \Delta g_{f}=O\left(e^{\vartheta T_{0}}\right)$ and is negative. From this it follows $P_{u_{2} u_{1} w_{1}}^{\varepsilon}\left(T_{0}^{\varepsilon}\right)=O\left(e^{\vartheta T_{0}}\right)$ and is negative. Similarly both $P_{u_{2} u_{1} w_{2}}^{\varepsilon}\left(T_{0}^{\varepsilon}\right)$ and $P_{u_{1} w_{1} w_{2}}^{\varepsilon}\left(T_{0}^{\varepsilon}\right)$ are $O\left(e^{\vartheta T_{0}}\right)$ and negative. At $\xi=T_{0}, P_{u_{2} w_{2} \vartheta}^{f}\left(T_{0}\right)=$ $-w_{2}\left(\partial / \partial u_{1}\right) \Delta h_{f}=O(1)$. Therefore $P_{u_{2} w_{2} \vartheta}^{\varepsilon}\left(T_{0}^{\varepsilon}\right)=O(1)$. In fact any 3 -form $P_{a b \vartheta}^{\varepsilon}\left(T_{0}^{\varepsilon}\right)$ with $a, b \neq v_{1}$ or $v_{2}$ is $O(1)$. Lastly, at $\xi=T_{0}, P_{u_{2} v_{2} \vartheta}^{f}\left(T_{0}\right)=0$. Thus $P_{u_{2} v_{2} \vartheta}^{\varepsilon}\left(T_{0}^{\varepsilon}\right)=O(\varepsilon)$. Similarly, any 3 -form $P_{a b v_{i}}^{\varepsilon}\left(T_{0}^{\varepsilon}\right)$ with $v_{i}=v_{1}$ or $v_{2}$ is $O(\varepsilon)$. We summarize below. 


$$
\begin{aligned}
& P_{u_{2} w_{2} \vartheta}^{\varepsilon}\left(T_{0}^{\varepsilon}\right)=O(1) \quad, \quad P_{u_{2} v_{1} w_{2}}^{\varepsilon}\left(T_{0}^{\varepsilon}\right)=O(\varepsilon) \quad, \quad P_{u_{2} w_{1} w_{2}}^{\varepsilon}\left(T_{0}^{\varepsilon}\right)=O\left(e^{\vartheta T_{0}}\right) \\
& P_{u_{2} w_{1} \vartheta}^{\varepsilon}\left(T_{0}^{\varepsilon}\right)=O(1) \quad, \quad P_{u_{2} w_{1} v_{2}}^{\varepsilon}\left(T_{0}^{\varepsilon}\right)=O(\varepsilon) \quad, \quad P_{u_{1} w_{1} w_{2}}^{\varepsilon}\left(T_{0}^{\varepsilon}\right)=O\left(e^{\vartheta T_{0}}\right) \\
& P_{u_{2} u_{1} \vartheta}^{\varepsilon}\left(T_{0}^{\varepsilon}\right)=O(1) \quad, \quad P_{u_{2} v_{2} \vartheta}^{\varepsilon}\left(T_{0}^{\varepsilon}\right)=O(\varepsilon) \quad, \quad P_{u_{2} u_{1} w_{1}}^{\varepsilon}\left(T_{0}^{\varepsilon}\right)=O\left(e^{\vartheta T_{0}}\right) \\
& P_{w_{2} w_{1} \vartheta}^{\varepsilon}\left(T_{0}^{\varepsilon}\right)=O(1) \quad, \quad P_{u_{2} v_{1} \vartheta}^{\varepsilon}\left(T_{0}^{\varepsilon}\right)=O(\varepsilon) \quad, \quad P_{u_{2} u_{1} w_{2}}^{\varepsilon}\left(T_{0}^{\varepsilon}\right)=O\left(e^{\vartheta T_{0}}\right)
\end{aligned}
$$

One of the central aspects of the Exchange Lemma is that certain forms that are initially small remain small as they pass by a slow manifold. In [28], the smallness required is exponential. This can be achieved here by normalizing by $P_{u_{2} w_{1} w_{2}}^{\varepsilon}$, using the rule $\hat{P}_{a b c}=P_{a b c} /\left|P_{u_{2} w_{1} w_{2}}^{\varepsilon}\right|$. Thus, forms previously of $O(\varepsilon)$ and $O(1)$ are now exponentially small at $T_{0}^{\varepsilon}$ and the other relevant forms are $O(1)$. The evolution equation of interest is

$$
\begin{aligned}
\hat{P}_{u_{2} w_{1} w_{2}}^{\varepsilon}=2 \vartheta \hat{P}_{u_{2} w_{1} w_{2}}^{\varepsilon} & -\frac{\partial^{2} F}{\partial u_{1}^{2}} \hat{P}_{u_{2} u_{1} w_{2}}^{\varepsilon}+\frac{\partial^{2} F}{\partial u_{1} \partial u_{2}} \hat{P}_{u_{2} u_{1} w_{1}}^{\varepsilon} \\
- & w_{1} \hat{P}_{u_{2} w_{2} \vartheta}^{\varepsilon}+w_{2} \hat{P}_{u_{2} w_{1} \vartheta}^{\varepsilon}+\hat{P}_{u_{2} v_{1} w_{2}}^{\varepsilon}+\hat{P}_{u_{2} w_{1} v_{2}}^{\varepsilon}
\end{aligned}
$$

Using the Exchange Lemma, or by computing evolution equations for and using the estimates above, $\hat{P}_{u_{2} w_{2} \vartheta}^{\varepsilon}, \hat{P}_{u_{2} w_{1} \vartheta}^{\varepsilon}, \hat{P}_{u_{2} v_{1} w_{2}}^{\varepsilon}$ and $\hat{P}_{u_{2} w_{1} v_{2}}^{\varepsilon}$ are exponentially small on $\left[T_{0}^{\varepsilon}, T_{1}^{\varepsilon}\right]$. Thus, (38) reduces to

$$
\hat{P}_{u_{2} w_{1} w_{2}}^{\varepsilon}=2 \vartheta \hat{P}_{u_{2} w_{1} w_{2}}^{\varepsilon}-\frac{\partial^{2} F}{\partial u_{1}^{2}} \hat{P}_{u_{2} u_{1} w_{2}}^{\varepsilon}+\frac{\partial^{2} F}{\partial u_{1} \partial u_{2}} \hat{P}_{u_{2} u_{1} w_{1}}^{\varepsilon}+O\left(e^{-\vartheta T_{0} / \varepsilon}\right)
$$

The evolution equations for $\hat{P}_{u_{1} w_{1} w_{2}}^{\varepsilon}, \hat{P}_{u_{2} u_{1} w_{2}}^{\varepsilon}$ and $\hat{P}_{u_{2} u_{1} w_{1}}^{\varepsilon}$ are

$$
\begin{aligned}
& \hat{P}^{\prime}{ }_{u_{1} w_{1} w_{2}}^{\varepsilon}=\vartheta \hat{P}_{u_{1} w_{1} w_{2}}^{\varepsilon}-\frac{\partial^{2} F}{\partial u_{2}^{2}} \hat{P}_{u_{2} u_{1} w_{1}}^{\varepsilon}+\frac{\partial^{2} F}{\partial u_{2} \partial u_{1}} \hat{P}_{u_{2} u_{1} w_{2}}^{\varepsilon} \\
& +w_{2} \hat{P}_{u_{1} w_{1} \vartheta}^{\varepsilon}-w_{1} \hat{P}_{u_{1} w_{2} \vartheta}^{\varepsilon}+\hat{P}_{u_{1} w_{1} v_{2}}^{\varepsilon}+\hat{P}_{u_{1} v_{1} w_{2}}^{\varepsilon}, \\
& \hat{P}^{\prime \varepsilon}{ }_{u_{2} u_{1} w_{2}}^{\varepsilon}=\vartheta \hat{P}_{u_{2} u_{1} w_{2}}^{\varepsilon}+\hat{P}_{u_{2} w_{1} w_{2}}^{\varepsilon}+w_{2} \hat{P}_{u_{2} u_{1} \vartheta}^{\varepsilon}+\hat{P}_{u_{2} u_{1} v_{2}}^{\varepsilon}, \\
& \hat{P}^{\prime \varepsilon}{ }_{u_{2} u_{1} w_{1}}^{\varepsilon}=\vartheta \hat{P}_{u_{2} u_{1} w_{1}}^{\varepsilon}+\hat{P}_{u_{1} w_{1} w_{2}}^{\varepsilon}+w_{1} \hat{P}_{u_{2} u_{1} \vartheta}^{\varepsilon}+\hat{P}_{u_{2} u_{1} v_{1}}^{\varepsilon} \text {, }
\end{aligned}
$$

which, as above, reduce to 


$$
\begin{aligned}
\hat{P}^{\prime \varepsilon}{ }_{u_{1} w_{1} w_{2}} & =\vartheta \hat{P}_{u_{1} w_{1} w_{2}}^{\varepsilon}-\frac{\partial^{2} F}{\partial u_{2}^{2}} \hat{P}_{u_{2} u_{1} w_{1}}^{\varepsilon}+\frac{\partial^{2} F}{\partial u_{2} \partial u_{1}} \hat{P}_{u_{2} u_{1} w_{2}}^{\varepsilon}+O\left(e^{-\vartheta T_{0} / \varepsilon}\right), \\
\hat{P}_{u_{2} u_{1} w_{2}}^{\varepsilon} & =\vartheta \hat{P}_{u_{2} u_{1} w_{2}}^{\varepsilon}+\hat{P}_{u_{2} w_{1} w_{2}}^{\varepsilon}+O\left(e^{-\vartheta T_{0} / \varepsilon}\right), \\
\hat{P}^{\prime \varepsilon}{ }_{u_{2} u_{1} w_{1}}^{\varepsilon} & =\vartheta \hat{P}_{u_{2} u_{1} w_{1}}^{\varepsilon}+\hat{P}_{u_{1} w_{1} w_{2}}^{\varepsilon}+O\left(e^{-\vartheta T_{0} / \varepsilon}\right) .
\end{aligned}
$$

Note that on manifold (E) because of hypothesis $(\mathrm{H} 1),\left(\partial^{2} F / \partial u_{1}^{2}\right)<0$ and $\left(\partial^{2} F / \partial u_{2}^{2}\right)<0$. Also, by hypothesis $(\mathrm{H} 7),\left(\partial^{2} F / \partial u_{2} \partial u_{1}\right)>0$. Therefore at $\xi=T_{0}^{\varepsilon}, \hat{P}^{\prime}{ }_{u_{2} w_{1} w_{2}}^{\varepsilon}\left(T_{0}^{\varepsilon}\right)<0, \hat{P}^{\prime}{ }_{u_{1} w_{1} w_{2}}^{\varepsilon}\left(T_{0}^{\varepsilon}\right)<0, \hat{P}^{\prime \varepsilon}{ }_{u_{2} u_{1} w_{2}}\left(T_{0}^{\varepsilon}\right)<0$ and $\hat{P}^{\prime}{ }_{u_{2} u_{1} w_{1}}^{\varepsilon}\left(T_{0}^{\varepsilon}\right)<0$. Moreover, the 3 -form $\hat{P}_{u_{2} w_{1} w_{2}}^{\varepsilon}$ will continue to remain negative as long as $\hat{P}_{u_{1} w_{1} w_{2}}^{\varepsilon}, \hat{P}_{u_{2} u_{1} w_{2}}^{\varepsilon}$ and $\hat{P}_{u_{2} u_{1} w_{1}}^{\varepsilon}$ stay negative. At $T_{0}^{\varepsilon}$ all of these forms are negative. So renormalize the forms again such that at $T_{0}^{\varepsilon}$ the largest of the forms is less than -1 . Let

$$
M=\left\{\left(\hat{P}_{u_{2} w_{1} w_{2}}^{\varepsilon}, \hat{P}_{u_{1} w_{1} w_{2}}^{\varepsilon}, \hat{P}_{u_{2} u_{1} w_{2}}^{\varepsilon}, \hat{P}_{u_{2} u_{1} w_{1}}^{\varepsilon}\right) \quad: \quad \begin{array}{l}
\hat{P}_{u_{2} w_{1} w_{2}}^{\varepsilon} \leq-1, \hat{P}_{u_{1} w_{1} w_{2}}^{\varepsilon} \leq-1 \\
\\
\left.\hat{P}_{u_{2} u_{1} w_{2}}^{\varepsilon} \leq-1, \hat{P}_{u_{2} u_{1} w_{1}}^{\varepsilon} \leq-1\right\}
\end{array}\right.
$$

Using (38) and (39), it can be seen that $M$ is positively invariant . Moreover at $T_{0}^{\varepsilon},\left(\hat{P}_{u_{2} w_{1} w_{2}}^{\varepsilon}, \hat{P}_{u_{1} w_{1} w_{2}}^{\varepsilon}, \hat{P}_{u_{2} u_{1} w_{2}}^{\varepsilon}, \hat{P}_{u_{2} u_{1} w_{1}}^{\varepsilon}\right) \in M$. Therefore, these forms remain negative until at least $T_{1}^{\varepsilon}$. In particular $\hat{P}_{u_{2} w_{1} w_{2}}^{\varepsilon}\left(T_{1}^{\varepsilon}\right)<0$ and, equivalently, $P_{u_{2} w_{1} w_{2}}^{\varepsilon}\left(T_{1}^{\varepsilon}\right)<0$.

As mentioned earlier, $P_{u_{2} w_{1} w_{2}}^{b}>0$ along the back. So $k_{1}<0$. Thus using equation (34), an application of Gronwall's Inequality and a possible renormalization of forms, we obtain

$$
P_{u_{2} w_{1} w_{2}}^{\varepsilon}\left(\tau_{\varepsilon}\right)=-P_{u_{2} w_{1} w_{2}}^{b}(\tau)+O(\varepsilon)
$$

In particular, $\operatorname{sgn} P_{u_{2} w_{1} w_{2}}^{\varepsilon}\left(\tau_{\varepsilon}\right)=-\operatorname{sgn} P_{u_{2} w_{1} w_{2}}^{b}(\tau)<0$. This concludes the proof of Lemma 4.3 .

Using (33) and Lemma 4.3, we obtain $D^{\prime}(0)>0$ as desired, concluding the proof of Theorem 4.1.

\section{References}

[1] J. Alexander, R. Gardner and C. Jones, A topological invariant arising in the stability analysis of travelling waves, J. Reine Angew. Math. 410 (1990), 167-212.

[2] J. Alexander and C. Jones, Existence and stability of asymptotically oscillatory double pulses, J. Reine Angew. Math. 446 (1994), 49-79.

[3] V. I. ARNoL'D, Characteristic class entering in quantization conditions, Funct. Anal. Appl. 1 (1967), 1-14. 
[4] V. I. Arnol'D, The sturm theorem and symplectic geometry, Funct. Anal. Appl. 19 (1985), 251-259.

[5] P.W. Bates AND C.K.R.T. Jones, Invariant manifolds for semilinear partial differential equations, Dynamics Reported 2 (1988).

[6] A. Bose, Existence and stability of travelling waves for coupled nerve axon equations, PhD thesis, Brown University (1994).

[7] A. Bose, Symmetric and anti-symmetric pulses in parallel coupled nerve fibres, to appear SIAM J. Appl. Math., 1995.

[8] R. Вотт, On the iterations of closed geodesics and the Sturm intersection theory, Commun. Pure Appl. Math. 9 (1956), 171-206.

[9] G. Carpenter, A geometric approach to singular perturbation problems with applications to nerve impulse equations, J. Diff. Equ. 23 (1977), 335-367.

[10] R. Casten, H. Cohen, P. Lagerstrom, Perturbation analysis of an approximation to the Hodgkin-Huxley Theory, Quart. Appl. Math., 32 (1975), 365-402.

[11] C. Conley, An oscillation theorem for linear systems with more than one degree of freedom, IBM Technical Report \#18004. IBM Watson Research Center, Yorktown Heights, New York (1972).

[12] J. Dockery, Existence and stability of travelling waves for parallel excitable fibers, in preparation

[13] J. W. Evans, Nerve axon equations, I: Linear approximations, Indiana Univ. Math J. 21 (1972), 877-955.

[14] J. W. Evans, Nerve axon equations, II: Stability at rest, Indiana Univ. Math J. 22 (1972), 75-90.

[15] J. W. Evans, Nerve axon equations, III: Stability of the nerve impulse, Indiana Univ. Math J. 22 (1972), 577-594.

[16] J. W. Evans, Nerve axon equations, IV: The stable and unstable impulse, Indian Univ. Math J. 24 (1975), 1169-1190.

[17] N. Fenichel, Persistence and smoothness of invariant manifolds for flows, Indiana Univ. Math J. 21 (1971), 193-226.

[18] P. Fife AND J.B. MCLeOd, The approach of solutions of nonlinear diffusion equations to travelling front solutions, Arch. Rational Mech. Anal. 65 (1977), 335-361.

[19] R. FitzHugh, Impulses and physiological states in theoretical models of nerve membranes, Biophys. J. 1 (1961), 445-466.

[20] R. A. Gardner and C.K.R.T. Jones, Travelling waves of a perturbed diffusion equation arising in a phase field model, Indiana Univ. Math J. 38 (1989), 1197-1222.

[21] R. A. Gardner and C.K.R.T. Jones, A stability index for steady state solutions of boundary value problems for parabolic systems, J. Diff. Eq. (2) 91, (1991), 181-203.

[22] R. A. Gardner and C.K.R.T. Jones, Stability of travelling wave solutions of diffusive predator-prey systems, Trans. AMS 327 No. 2 (1991), 465-524.

[23] S. P. Hastings, On the existence of homoclinic and periodic orbits for the FitzHugh-Nagumo equations, Quart. J. Math. Oxford Ser. (2) 27 (1976), 123-134.

[24] D. Henry, The geometric theory of semilinear parabolic equations, Lect. Notes in Math. 840, Berlin 1981.

[25] A.L. Hodgkin and A.F. Huxley, A quantitative description of membrane current and its application to conduction and excitation in nerve, J. Physiol. 117 (1952d), 500-544.

[26] C. K. R. T. Jones, Stability of the travelling wave solution of the FitzHughNagumo system, Trans. AMS 286, No. 2 (1984), 431-469. 
[27] C. K. R. T. Jones, Instability of standing waves for non-linear Schrödingertype equations, Ergod. Th. \& Dynam. Sys. 8* (1988), 119-138.

[28] C. K. R. T. Jones and N. KopelL, Tracking invariant manifolds with differential forms in singularly perturbed systems, J. Diff. Eq. 108 No.1 (1994), 64-88.

[29] C. K. R. T. Jones, N. Kopell and R. Langer, Construction of the FitzHughNagumo pulse using differential forms, in Patterns and Dynamics in Reactive Media, IMA volumes in mathematics and its applications, 37, H. Swinney, G. Aris and D. Aronson eds, Springer-Verlag, NY, (1991) 101116.

[30] J. Keener, Frequency decoupling of parallel excitable fibers, SIAM J. Appl. Math. 49 (1989), 211-230.

[31] W. Massey, A basic course in Algebraic Topology, Springer-Verlag, NY 1991.

32. J. Milnor, Morse Theory, Princeton University Press, 511963.

[33] J. Nagumo, S. Arimoto and S. Yoshizawa, An active impulse transmission line simulating nerve axons, Proc. IRL 50 (1960), 2061-2070.

[34] A. Pazy, Semigroups of Linear Operators and Applications to Partial Differential Equations, Berlin 1983.

[35] D. Sattinger, On the stability of waves of nonlinear parabolic systems, Adv. in Math. 22 (1976), 312-355.

[36] A. Scott and S. Luzader, Coupled solitary waves in neurophysics, Phys. Script. 20 (1979), 395-491.

[37] E. YANAGida, Stability of fast travelling pulse solutions of the FitzHughNagumo equations, J. Math. Biol. 22 (1985), 81-104.

Research of both authors supported in part by NSF Grant DMS-9100085. The second author was also supported by an award from the Alexander von Humboldt Foundation.

Amitabha Bose

Department of Mathematics

Boston University

Boston, Massachusetts 02215

Christopher K. R. T. Jones

Division of Applied Mathematics

Brown University

Providence, Rhode Island 02912-9106

Received: August 8th, 1994; revised: February 24th, 1995. 